$11-2019$

\title{
Alterations in Phenotype and Gene Expression of Adult Human Aneurysmal Smooth Muscle Cells by Exogenous Nitric Oxide
}

\author{
Kurt Farrell \\ Cleveland State University \\ Phillip Simmers \\ Cleveland State University \\ Gautam Mahajana \\ Cleveland State University \\ Ludovic Boytard \\ University of Lille \\ Andrew Camardo \\ Cleveland Clinic \\ Follow this and additional works at: https://engagedscholarship.csuohio.edu/encbe_facpub \\ Sre next page for additional authors \\ Part of the Chemical Engineering Commons \\ How does access to this work benefit you? Let us know!
}

\section{Repository Citation}

Farrell, Kurt; Simmers, Phillip; Mahajana, Gautam; Boytard, Ludovic; Camardo, Andrew; Joshi, Jyotsna; Ramamurthi, Anand; Pine, Florence; and Kothapalli, Chandrasekhar R., "Alterations in Phenotype and Gene Expression of Adult Human Aneurysmal Smooth Muscle Cells by Exogenous Nitric Oxide" (2019).

Chemical \& Biomedical Engineering Faculty Publications. 165.

https://engagedscholarship.csuohio.edu/encbe_facpub/165

This Article is brought to you for free and open access by the Chemical \& Biomedical Engineering Department at EngagedScholarship@CSU. It has been accepted for inclusion in Chemical \& Biomedical Engineering Faculty Publications by an authorized administrator of EngagedScholarship@CSU. For more information, please contact library.es@csuohio.edu. 


\section{Authors}

Kurt Farrell, Phillip Simmers, Gautam Mahajana, Ludovic Boytard, Andrew Camardo, Jyotsna Joshi, Anand Ramamurthi, Florence Pine, and Chandrasekhar R. Kothapalli 


\title{
Alterations in phenotype and gene expression of adult human aneurysmal smooth muscle cells by exogenous nitric oxide
}

\author{
Kurt Farrell, Phillip Simmers, Gautam Mahajan, Ludovic Boytard, Andrew Camardo, Jyotsna \\ Joshi, Anand Ramamurthi, Florence Pinet, Chandrasekhar R. Kothapalli
}

A R T I C L E I N F O

Keywords:

Abdominal aortic aneurysm

Elastin

Matrix proteins

Cell modulus

Matrix metalloproteinases

Nitric oxide

LILAS study

\begin{abstract}
A B S T R A C T
Abdominal aortic aneurysms (AAA) are characterized by matrix remodeling, elastin degradation, absence of nitric oxide (NO) signaling, and inflammation, influencing smooth muscle cell (SMC) phenotype and gene expression. Little is known about the biomolecular release and intrinsic biomechanics of human AAA-SMCs. NO delivery could be an attractive therapeutic strategy to restore lost functionality of AAA-SMCs by inhibiting inflammation and cell stiffening. We aim to establish the differences in phenotype and gene expression of adult human AAA-SMCs from healthy SMCs. Based on our previous study which showed benefits of optimal NO dosage delivered via S-Nitrosoglutathione (GSNO) to healthy aortic SMCs, we tested whether such benefits would occur in AAA-SMCs. The mRNA expression of three genes involved in matrix degradation (ACE, ADAMTS5 and ADAMTS8) was significantly downregulated in AAA-SMCs. Total protein and glycosaminoglycans synthesis were higher in AAA-SMCs than healthy-SMCs ( $p<0.05$ for AAA-vs. healthy- SMC cultures) and was enhanced by GSNO and 3D cultures ( $p<0.05$ for 3D vs. 2D cultures; $p<0.05$ for GSNO vs. non-GSNO cases). Elastin gene expression, synthesis and deposition, desmosine crosslinker levels, and lysyl oxidase (LOX) functional activity were lower, while cell proliferation, iNOS, LOX and fibrillin-1 gene expressions were higher in AAA-SMCs ( $p<0.05$ between respective cases), with differential benefits from GSNO exposure. GSNO and 3D cultures reduced MMPs $-2,-9$, and increased TIMP-1 release in AAA-SMC cultures $(p<0.05$ for GSNO vs. non-GSNO cultures). AAA-SMCs were inherently stiffer and had smoother surface than healthy SMCs $(p<0.01$ in both cases), but GSNO reduced stiffness $(\sim 25 \% ; p<0.01)$ and increased roughness $(p<0.05)$ of both cell types. In conclusion, exogenously-delivered NO offers an attractive strategy by providing therapeutic benefits to AAASMCs.
\end{abstract}

\section{Introduction}

Chronic vascular pathological conditions (e.g., hypertension, atherosclerosis, trauma, infection) promote localized release of proteinases and enzymes which break down the intact extracellular matrix (ECM; e.g., elastin, glycosaminoglycans (GAGs)) within the adult abdominal aortic walls $[1,2]$. Unfortunately, the major cell types in aorta, i.e., smooth muscle cells (SMCs) and adventitial fibroblasts, are limited in their ability to repair, replace or regenerate the degraded vascular matrix, which often results in progressive weakening and burgeoning of blood vessel diameter ( $>50 \%$ increase relative to healthy vessel) - a condition clinically termed as abdominal aortic aneurysm (AAA). AAAs are also typically characterized by the presence of innate and adaptive immune cells (e.g., macrophages, T-cells, neutrophils, lymphocytes) which release abnormal levels of cytokines and matrix metalloproteinases (MMPs) to exacerbate the breakdown of elastic fibers and other matrix proteins $[3,4]$. This, in turn, disrupts the signaling between elastic fibers and SMCs, promotes SMC apoptosis, and weakens elasticity in blood vessel walls, to permanently alter the mechanical properties and native microenvironment of the vessel wall $[5,6]$.

Among the major ECM components native to blood vessels, collagen, GAGs and elastin play an important role in regulating cell adhesion, anchoring, proliferation, and migration [4,7]. They also regulate blood vessel homeostasis by supporting axial and circumferential 


\section{List of abbreviations}

(AAA) abdominal aortic aneurysms

(ACE) angiotensin-converting enzyme

(ADAMTS) A disintegrin and metalloproteinase with thrombospondin motifs

(AFM) atomic force microscope

(ECM) extracellular matrix

(E) elastic modulus

(ELN) elastin gene

(FBN) fibrillin gene

(GAGs) glycosaminoglycans
(HA) hyaluronan

(Hu-ECs) human endothelial cells

(iNOS) inducible nitric oxide synthase

(LOX) lysyl oxidase

(MMP) matrix metalloproteinases

(NO) nitric oxide

(NSAIDs) non-steroidal anti-inflammatory drugs

(SMC) smooth muscle cell

(GSNO) S-Nitrosoglutathione

( $\alpha$-SMA) $\alpha$-smooth muscle actin

(TIMP) tissue inhibitors of metalloproteinases loadings and conferring anti-thrombogenic properties [8]. These ECM molecules are synthesized, crosslinked, and deposited as mature matrix fibers by SMCs and adventitial fibroblasts within the aorta. While the turnover of collagens and GAGs is maintained even through adolescence, elastic matrix fiber deposition primarily occurs only during developmental stages and preserved throughout the adult life (elastin half-life is $\sim 74$ years) [9]. Since healthy elastic fibers play critical biochemical and biomechanical roles in maintaining vessel homeostasis, abnormalities in elastin formation due to genetic defects or elastin degradation due to diseases could result in peptides formation, which obfuscates the beneficial role of intact elastic fibers [10,11].

Given the limitations of existing surgical (e.g., endovascular aneurysm repair, open surgery), pharmacological (e.g., anti-inflammation agents, beta-blockers, statins) and preservation (e.g., MMPs inhibition, TIMPs delivery, elastases suppression) interventions for comprehensive AAA treatment [9,12-15], ECM (specifically elastin) repair and regeneration approaches focusing on tissue-engineered grafts, stem cells, 3D scaffolds, bimolecular cues, biomechanics, and their combinations, are being explored $[16,17]$. While the phenotype and gene expression of ECM proteins synthesized by healthy Hu-SMCs has been reported [18-21], similar outcomes from their AAA-counterparts are unknown. Specifically, the baseline levels of matrix proteins synthesized and deposited by human AAA-SMCs, changes in their phenotype and gene expression, the cytokines and chemokines released, their stiffness levels, and the responses to exogenous biomolecular cues, remains unexplored. Such information is critical for understanding the disease etiology and identify effective pharmacological, tissue engineering and regenerative medicine strategies to repair and regenerate elastin in AAA vessels.

We have recently reported on the benefits of delivering exogenous nitric oxide (NO) cues via S-nitrosoglutathione (GSNO), to cell proliferation and ECM deposition by adult human aortic SMCs (Hu-SMCs) within 3D biomimetic cocultures with adult human endothelial cells (Hu-ECs) [22]. Such NO delivery from GSNO was also shown to suppress cell proliferation and stimulate mRNA expression and synthesis of tropoelastin and lysyl oxidase (LOX) in chick aortic SMC cultures [23]. Physiologically, NO is produced from L-arginine and oxygen by endothelium nitric oxide synthases (eNOS) [24], and exogenous NO
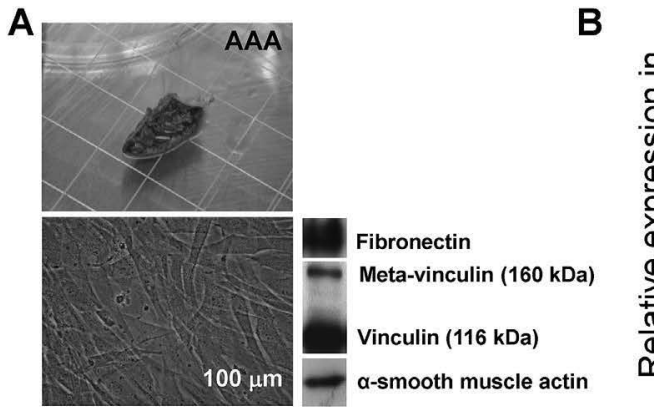

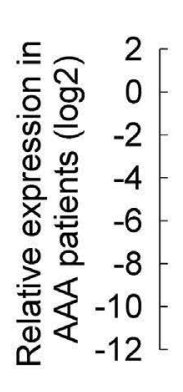

ACE

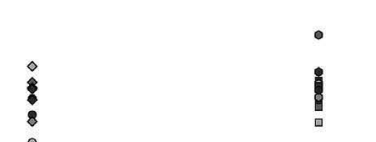
$\begin{array}{lll}\text { ADAMTS8 ADAMTS5 } & \text { Fig. 1. (A) A sample AAA tissue }(n=20 \text { patients) } \\ \text { used for SMC cultures. Representative phase-contrast }\end{array}$ microscopy of SMCs obtained by explant from human AAA biopsies. Western blot analysis of SMC for fibronectin, meta-vinculin $(160 \mathrm{kDa})$, vinculin $(116 \mathrm{kDa})$ and $\alpha$-smooth muscle actin ( $\alpha$-SMA). Scale bar: $100 \mu \mathrm{m}$. (B) mRNA expression ratio of genes ( $2^{-\mathrm{dCt}}$; normalized to house-keeping genes) in SMCs derived from AAA $(n=12)$ patients, potentially involved in matrix degrading processes in AAA, as determined by RT-PCR.
LILAS was a case-control study that enrolled 42 men, some with
AAA, who needed a vascular surgery or endovascular treatment at the
Lille University Hospital Centre (Lille, France) between September
2003 and July 2005 [33]. Case patients were considered eligible if the
AAA diameter, measured by abdominal ultrasound exceeded $50 \mathrm{~mm}$, or
Fig. 1. (A) A sample AAA tissue ( $n=20$ patients)

LILAS was a case-control study that enrolled 42 men, some with
AAA, who needed a vascular surgery or endovascular treatment at the
Lille University Hospital Centre (Lille, France) between September
2003 and July 2005 [33]. Case patients were considered eligible if the
AAA diameter, measured by abdominal ultrasound exceeded $50 \mathrm{~mm}$, or
Fig. 1. (A) A sample AAA tissue ( $n=20$ patients)

LILAS was a case-control study that enrolled 42 men, some with
AAA, who needed a vascular surgery or endovascular treatment at the
Lille University Hospital Centre (Lille, France) between September
2003 and July 2005 [33]. Case patients were considered eligible if the
AAA diameter, measured by abdominal ultrasound exceeded $50 \mathrm{~mm}$, or
Fig. 1. (A) A sample AAA tissue ( $n=20$ patients)

LILAS was a case-control study that enrolled 42 men, some with
AAA, who needed a vascular surgery or endovascular treatment at the
Lille University Hospital Centre (Lille, France) between September
2003 and July 2005 [33]. Case patients were considered eligible if the
AAA diameter, measured by abdominal ultrasound exceeded $50 \mathrm{~mm}$, or
Fig. 1. (A) A sample AAA tissue ( $n=20$ patients)

exposure inhibits eNOS activity [25,26] and induces S-nitrosylation of cysteine residues [27]. NO donors and eNOS gene transfer were shown to inhibit SMC proliferation in vitro, resulting in a delay in cell cycle progression but not apoptosis [28-30]. NO delivered from the donor compounds such as diethylenetriamine NONOate and S-nitro-N-acetylpenicillamine inhibited elastase release and activity, and matrix remodeling by porcine pulmonary artery SMCs, by repressing ERK phosphorylation [31,32]. These studies attest to the role of NO donors and NOS in promoting elastogenesis at transcriptional- \& translationallevels, and attenuating inflammation within diseased aortic tissues.

This study is based on the hypothesis that exogenous NO delivery to AAA-SMCs will inhibit inflammatory markers release, suppress cell proliferation, promote elastic matrix synthesis, and enhance healthy and contractile phenotype, thereby providing an attractive therapeutic strategy to restore the lost functionality of AAA-SMCs and improve vascular tone. We first investigated the changes in transcription levels of AAA-SMC genes, involved in the pathological progression of this disease. Next, we assessed the in vitro baseline levels of cell proliferation, phenotypic and genetic expression, ECM and biomolecular (elastin, sulfated glycosaminoglycans (sGAGs), hyaluronan (HA), LOX, desmosine, inducible nitric oxide synthase - iNOS) synthesis and deposition, release of cytokines/chemokines/MMPs/TIMPs, and biomechanical characteristics of human AAA-SMCs. Similar outcomes from healthy Hu-SMCs were assessed under identical culture conditions for comparison. Finally, the response of AAA-SMCs to exogenously-delivered NO cues was evaluated and compared to their healthy counterparts, to investigate the utility of delivering exogenous NO for AAA treatment.

\section{Materials and methods}

\subsection{Population study}


if it had increased more than $10 \mathrm{~mm}$ during the past six months and required initial surgical treatment (Supplementary Table 1). The study conformed to the principles outlined in the Declaration of Helsinki, ethics committee of the Lille University Hospital Centre (France) approved the study, and each patient provided written informed consent. Clinical data items were presented as mean $\pm \mathrm{SD}$, and frequencies with proportions as appropriate. Statistical significance was assigned at $p<0.05$.

\subsection{Isolation of human AAA-SMCs}

Aortic samples (Fig. 1A) were obtained for 20 of the 24 AAA patients and SMCs were isolated from all the 20 AAA tissue samples. Individual cultures of AAA-SMCs were established for each patient by dissection of a residual segment of abdominal aorta and then cutting out the media into 1-2 cu.mm pieces and culturing them on type 1 collagen coated dishes (rat tail-derived, BD Biosciences, France) in medium 231 supplemented with smooth muscle growth supplement (Cascade Biologics, France) and antibiotics $(50 \mu \mathrm{g} / \mathrm{mL}$ streptomycin, 50 $\mathrm{U} / \mathrm{mL}$ penicillin; Sigma-Aldrich). SMCs were characterized by Western blot analysis. Twenty microgram fractions of cell lysates were separated by SDS-PAGE ( $9 \%$ polyacrylamide), transferred to Hybond C membranes (GE Healthcare, France) and blotted with antibodies against $\alpha$ smooth muscle actin ( $\alpha$-SMA) (1A4, Dako, 1:5000), fibronectin (E3A, Sigma-Aldrich, 1:3000), and vinculin/meta-vinculin (hVIN-1, SigmaAldrich, 1:400). The blots were subsequently washed in PBS-Tween 20 and incubated with appropriate secondary antibodies (peroxidaseconjugated anti-mouse IgG, GE Healthcare) at a dilution of 1:5000. The blots were then washed three times in PBS-Tween 20 for $15 \mathrm{~min}$, incubated with enhanced chemiluminescence reagents (GE Healthcare), and exposed to a radiographic film and scanned with an Imagescanner ${ }^{\circledR}$ (GE Healthcare). AAA-SMCs were subsequently passaged with Medium 231 (Life Technologies).

\subsection{Transcriptomic analysis of human AAA-SMCs}

Changes in SMCs were validated for selected genes with qRT-PCR. RNA samples isolated from SMCs were converted to cDNA with an in vitro reverse transcription reaction. These cDNA were used as templates for Taqman qRT-PCR with ABI Assays-on-Demand on an ABI Prism 7900 sequence detection system. The specific assays were designed by TaqMan $^{\circledR}$ Gene Expression Assays (Applied Biosystems) and used Hs 00199841-m1 (ADAMTS 5), Hs 00199836-m1 (ADAMTS 8), Hs 00166915-m1 (renin), Hs 00174179-m1 (ACE1), and Hs 00377632-m1 (L-cathepsin). All samples were run in triplicate, with GADPH (Hs99999905_m1) and Cyclophilin A (Hs99999904_m1) as internal controls to normalize transcript abundance ( $2^{-\mathrm{dCt}}$ method). Triplicates were averaged to calculate an expression value for each sample.

\subsection{Human $A A A$ and healthy aortic $S M C$ cultures}

AAA-SMCs derived from one patient (details in Table 1) were investigated further using biochemical and biomechanical analysis to establish the baseline. Healthy adult abdominal aortic Hu-SMCs were obtained from Life Technologies Corp. (Carlsbad, CA, USA) and passaged using Medium 231 (ThermoFisher Scientific, USA). AAA-SMCs were cultured on 2D collagen-coated $(50 \mu \mathrm{g} / \mathrm{mL})$ substrates or within $3 \mathrm{D}$ collagen hydrogels $(2 \mathrm{mg} / \mathrm{mL} ; \mathrm{pH} \sim 7.2)$. Results from 2D substrates will help establish the baseline values, while cultures in 3D gels will provide more physiological-mimic microenvironment. Cells were cultured for 21 days in SMC media, containing $100 \mathrm{nM}$ GSNO (Sigma, Saint-Louis, MO, USA), a NO donor. Control cultures received no GSNO (termed $0 \mathrm{nM}$ ). The culture media contained recombinant human insulin-like growth factor-I $(2 \mu \mathrm{g} / \mathrm{mL})$ and glucose (D-Glucose; levels unknown). Healthy and AAA-derived SMCs were imaged at random locations during passaging using a Zeiss Axiovert A1 fluorescence microscope equipped with Hamamatsu camera and image acquisition software. At least ten images per cell type were processed using ImageJ (NIH) to quantify the perimeter and area of each cell type.

Two sets of cultures were performed in parallel. 3D collagen gels were prepared by mixing collagen stock solution (type 1 , rat-tail derived, BD Biosciences, Bedford, MA, USA) with a mixture of $10 \times$ PBS, $1 \mathrm{M} \mathrm{NaOH}$ and deionized water. For 3D cultures, SMCs were mixed in collagen gels at a density of $10^{4}$ cells per well in 48 -well plates. The cultures were placed in a humidified incubator $\left(37^{\circ} \mathrm{C}, 30 \mathrm{~min}\right)$ to polymerize the collagen gels. For 2D cultures, collagen-coated 24-well tissue culture plastic plates were seeded with $10^{4}$ cells per well. Both healthy- and AAA- SMCs were plated from passages 4-6. GSNO dose used in this study was identified from our previous study [22] where a range of GSNO doses were tested to evaluate their effects on ECM synthesis and deposition. Media was changed once every two days and the spent medium was pooled over the 21-day culture and stored at $-20^{\circ} \mathrm{C}$ for further biochemical analyses. Similarly, cell matrix layers at the end of 21 days were trypsinized and processed for biochemical assays.

\subsection{Biochemical assays}

At the end of 21 days, the amounts of various protein types released into the pooled media or deposited within cell matrix of $2 \mathrm{D}$ and $3 \mathrm{D}$ cultures were quantified as detailed earlier [22]. Individual biochemical assays were performed on AAA-SMC cultures to quantify total protein (BCA Kit Assay; Sigma-Aldrich), elastin (Fastin assay; Accurate Scientific Corp, Westbury, NY), sulfated glycosaminoglycans (sGAGs; Kamiya Biomedical Company, Seattle, WA), and HA (hyaluronan enzyme-linked immunosorbent quantitative assay; Echelon Biosciences Inc., Salt Lake City, UT) amounts. The protein amounts within cells was not separated from the total protein quantified within cell layers. The non-cellular protein contribution to the BCA assay (from collagen gels in which the cells were cultured) was accounted by estimating the protein content in $2 \mathrm{mg} / \mathrm{mL}$ collagen gels and collagen-coated 2D cultures without cells, and deducting from the measured total protein amounts reported in test cases. Although other ECM proteins such as collagens might be released and deposited by SMCs, their amounts were not assessed here. SMC proliferation was quantified using a fluorometric assay detailed earlier [22], to quantify the amount of DNA

Table 1

Baseline characteristics of the study population in AAA group. AAA: aortic abdominal aneurysm; CAD: coronary artery disease; MI: myocardial infarction; ACS: acute coronary syndrome; PTCA: percutaneous transluminal coronary angioplasty. Data expressed as mean $\pm \mathrm{SD}$, where appropriate.

$\begin{array}{ll}\text { Age, years } & 68.0 \pm 6.1 \\ \text { Male gender, \% } & 24(100) \\ \text { Anthropometric data } & \\ \text { Weight, kg } & 82.9 \pm 11.6 \\ \text { Height, cm } & 173.8 \pm 5.4 \\ \text { Body mass index, } \mathrm{kg} / \mathrm{m}^{2} & 27.4 \pm 3.7 \\ \text { Cardiovascular risk factors, n (\%) } & \\ \text { Current smoking } & 4(17) \\ \text { Past smoking } & 17(71) \\ \text { Hypercholesterolemia } & 14(58) \\ \text { Hypertension } & 15(63) \\ \text { Diabetes mellitus } & 4(17) \\ \text { Familial history of CAD } & 4(17) \\ \text { Personal history of CAD, n (\%) Stable } & \\ \text { Angina } & 2(8) \\ \text { MI or ACS } & 7(29) \\ \text { Coronary artery bypass } & 1(4) \\ \text { PTCA } & 4(17) \\ \text { History of stroke, n (\%) } & 0\end{array}$


synthesized over the 21 days. Similarly, LOX functional activity was quantified using a fluorometric assay (AmplexRed ${ }^{\circledR}$ Assay; Molecular Probes, Eugene, OR).

\subsection{Desmosine bioassay}

The amount of desmosine produced and released by AAA-SMCs and healthy SMCs into the pooled media was quantified using a sandwich enzyme immunoassay technique (Human Desmosine ELISA Kit; MyBioSource, Inc., San Diego, CA). The desmosine bioassay has a detection range of $0.1-10 \mathrm{ng} / \mathrm{mL}$ and a sensitivity of $0.039 \mathrm{ng} / \mathrm{mL}$. Briefly, aliquots of samples and standards were incubated in a 48-strip-well precoated with desmosine-specific antibody, and the bound desmosine was serially-tagged with biotin-conjugated DES-specific antibody and avidin-conjugated horseradish peroxidase, with intermediate washings after every step. Finally, desmosine amounts in each sample were assessed calorimetrically based on the intensity of color generated within a substrate solution in proportion to the respective DES amounts.

\subsection{Real-time PCR analysis of SMCs}

For RT-PCR analysis, AAA-SMCs and healthy SMCs were cultured in parallel on 2D collagen-coated substrates or within 3D collagen gels and exposed to $100 \mathrm{nM}$ GSNO for 5 days to induce changes in gene expression, and GSNO-free cultures served as controls. Total RNA was extracted from SMCs using RNAqueous ${ }^{\circledR}$-Micro total RNA isolation kit (Life technologies) according to the manufacturer's instructions. The RNA concentration of each sample was determined using a NanoDrop ND-1000 spectrophotometer (Thermo Scientific, Wilmington, DE). The RNA was reverse-transcribed to cDNA using the high-capacity cDNA reverse transcription kit with RNase inhibitor (Life Technologies) according to manufacturer's instructions. Gene expression in SMCs was quantified using TaqMan ${ }^{\circledR}$ gene expression assays (Applied Biosystems), and TaqMan ${ }^{\circledast}$ Gene expression master mix for each target using the ABI 7500 Real-time PCR System (Applied Biosystems, Forest City CA) limited to only 40 cycles. The following genes (details in Supplementary Table 2) were analyzed: lysyl oxidase (LOX, Assay ID: Hs00942480_m1), elastin (ELN, Assay ID: Hs00355783_m1), fibrillin 1 (FBN1, Assay ID: Hs00171191_m1), and inducible nitric oxide synthase 2 (NOS2, Assay ID: Hs01075529_m1). To identify relative differences in the levels of target genes, gene expression in AAA-SMCs was normalized to endogenous reference gene (18S, Assay ID: 4319413E) and further to corresponding expression in healthy SMCs, using the $\Delta \Delta \mathrm{Ct}$ method.

\subsection{Cytokine/chemokine assays}

Cytokine, chemokine, TIMP and MMP analyses were performed using Discovery Assays ${ }^{\circledast}$ (Eve Technologies, Alberta Canada), from 2D or 3D cultures of AAA-SMCs and healthy SMCs. Briefly, cell culture supernatants were collected from each well after $24 \mathrm{~h}$, spun down at $3000 \mathrm{~g}$ for $5 \mathrm{~min}$, and stored at $-20^{\circ} \mathrm{C}$. The supernatants were then processed using multiplexing LASER bead technology and processed on a dual-laser flow-cytometry system (Bio-Plex 200). The technology works by utilizing different combinations of red and infrared fluorophore beads conjugated to specific antibodies targeted to the cytokine or chemokine of choice and read using a flow-cytometry based system. The quantity of the specific analyte generated was based off a series of standards set forth by the company. The assays performed include human focused 11-plex, human MMP 9-plex and TIMP 4-plex, to quantify the following analytes: GM-CSF, IFN- $\gamma$, IL-1 $\beta$, IL-2, IL-4, IL-6, IL-8, IL-10, IL-12 (p70), MCP-1, TNF- $\alpha$, MMPs-1, 2, 3, 7, 8, 9, 10, 12, 13, and TIMPs-2, 3, 4.

\subsection{Western blot analysis of iNOS}

iNOS expression within healthy and AAA-SMCs, in both 2D and 3D cultures, in the presence or absence of GSNO, was semi-quantitatively evaluated using Western blot analysis as we detailed elsewhere [22]. To optimize loading sample volumes for SDS/PAGE Western blot, cell pellets from respective cultures were assayed using a DC protein assay kit (Bio-Rad Labs). The protein bands were detected using a primary polyclonal antibody for the iNOS protein (Abcam), using SMC-alpha actin (Abcam) as the loading control, and the bands were visualized using chemiluminescence method and quantified using ImageJ.

\subsection{TIMP-1 assay}

TIMP-1 amounts released by healthy and AAA-SMCs into the pooled media, in the presence or absence of GSNO, were quantified using Human TIMP-1 Quantikine ELISA Kit (R\&D Systems, Minneapolis, MN). Spent media (supernates) from the cell cultures in 2D or 3D were pooled and stored at $-20^{\circ} \mathrm{C}$ and loaded onto a 96-well polystyrene microplate coated with a monoclonal antibody specific for human TIMP-1. Post-incubation ( $2 \mathrm{~h}, \mathrm{RT}$ ) on a horizontal orbital shaker, the bound proteins were incubated with human TIMP-1 specific horseradish peroxidase-linked polyclonal antibody ( $1 \mathrm{~h}, \mathrm{RT}$ ), exposed to a chromogen solution containing tetramethylbenzidine for (30 min, RT, dark), and the reaction was stopped with $2 \mathrm{~N}$ sulfuric acid. Final solutions were measured using Epoch BioTek microplate spectrophotometer with absorbance at $450 \mathrm{~nm}$. This assay has a range of $0.2-10 \mathrm{ng} / \mathrm{mL}$ in cell culture supernates, with a sensitivity of $0.08 \mathrm{ng} / \mathrm{mL}$.

\subsection{Immunofluorescence labeling of cell layers}

AAA-SMCs were cultured on glass chamber slides $\left(10^{4}\right.$ cells/well $)$ for 21 days in the presence or absence of $100 \mathrm{nM}$ GSNO, and the cell layers were fixed in cold methanol, washed with PBS, blocked with PBS containing $5 \% \mathrm{v} / \mathrm{v}$ goat serum (ThermoFisher Scientific) and immunolabeled with rabbit anti-human polyclonal antibodies against elastin (1:100 v/v dilution, Abcam), fibrillin-1 (1:100 v/v dilution, Bioss Antibodies), fibulin-4 (1:100 v/v dilution, Santa Cruz Biotechnology), and fibulin-5 (1:100 v/v dilution, Santa Cruz Biotechnology). Cell layers were washed with PBS and labeled with Alexafluor 633 goat anti-rabbit secondary antibody; 1:1000 dilution, ThermoFisher Scientific). The cell layers were washed with PBS and mounted with Vectashield containing the nuclear stain 4',6-Diamidino2-Phenylindole, Dihydrochloride (DAPI; Vector Laboratories, Burlingame, CA). AAA-SMC layers treated with the secondary probe alone (no primary antibody) served as negative controls. Cell layers were imaged with a Leica SP8 confocal microscope (Leica Microsystems Inc, Buffalo Grove, IL).

\subsection{Atomic force microscopy (AFM) analysis}

Healthy and AAA-SMCs were cultured in 35-mm laminin-coated petri dishes and maintained at $37^{\circ} \mathrm{C}$ throughout live-cell nanoindentation assay, over a 60-min experiment each time, performed using a MFP-3D-Bio AFM (Asylum Research, Santa Barbara, CA) mounted on an inverted optical microscope (Nikon Eclipse Ti). Tip-less AFM cantilevers (model Arrow TL 1, Nano World, nominal spring constant $\sim 0.03 \mathrm{~N} / \mathrm{m}$ ) were modified by attaching a $4.5 \mu \mathrm{m}$ polystyrene bead using epoxy [34]. The spherical bead indenter reduces the possibility of destructive deformation of cell due to smaller local strains applied [35], compensates for the $x-y$ drift (usually $<1 \mu \mathrm{m}$ ), and yields higher spatially-averaged measurement with minimal variability, relative to conventional sharp-tip probes. The actual spring constant was determined from a force-distance curve and using thermal calibration method in a clean culture dish containing DMEM. Drifting minimization and thermal/mechanical equilibrium of AFM was achieved by submerging probe in cell medium for stabilization. For each experiment, at least 30 cells were randomly selected, indented between nuclei and cell edges, and 150-200 force curves were obtained 
at random locations on each cell in force-volume mode, at a rate of $0.25 \mathrm{~Hz}$ with approach/retraction velocity of $2.5 \mu \mathrm{m} / \mathrm{s}$. Using Hertz's contact model $\left[F=\left(4 E \sqrt{R \delta^{3}}\right) / 3\left(1-\mu^{2}\right)\right]$, Young's modulus was determined from these force curves, where $F$ is the indentation force, $E$ is the Young's modulus, $\mu$ is the Poisson's ratio ( 0.5 for cells), $R$ is radius of spherical bead attached to cantilever and $\delta$ is the indentation depth $(300-400 \mathrm{~nm})$ [35]. The analysis of force-indentation curves was automated using Igor Pro 6.37 software. The indentation data was analyzed assuming cells behave as linearly elastic at small strain regimes. The mean elastic modulus values were calculated for each cell from the elastic modulus heat maps generated over randomly-selected $90 \times 90 \mu \mathrm{m}^{2}$ area, and then averaged across multiple cells for each culture condition group. From this data, the mean surface roughness (Ra) of each cell - characterized as the arithmetic mean of the deviations in height from the line mean value, and the root mean square (Rq), were calculated [36]. Both $\mathrm{Ra}$ and $\mathrm{Rq}$ were obtained from randomly selected areas $\left(30 \times 30 \mu \mathrm{m}^{2}\right)$ on the cell membrane. A two-tailed Student's t-test was applied to all the pairs of samples (untreated-treated cells) for statistical analysis and $p<0.05$ were considered significant.

\subsection{Statistical analysis}

All biochemical data were obtained from three independent repeat experiments per case ( $\mathrm{n}=3$ wells/case/experiment) and analyzed using one-way ANOVA followed by Tukey's HSD post-hoc testing, assuming unequal variance and differences deemed significant for $p<0.05$. The data were assumed to follow a near-Gaussian distribution in all the cases, and the mean and standard errors were calculated accordingly.

\section{Results}

\subsection{Patients}

Table 1 summarizes the baseline characteristics of the 42 AAA subjects. One-third of the AAA patients had hypertension, half had dyslipidemia, $17 \%$ had diabetes mellitus, and $29 \%$ had family members who had a history of heart disease (myocardial infarction and/or acute coronary syndrome). The AAA case patients $(n=24)$ had a mean maximal external aortic diameter of $56.1 \pm 11.3 \mathrm{~mm}$ (range: 46-95 mm). Fewer AAA patients received ACE inhibitor treatment ( $21 \%$ vs. $44 \%$; Supplementary Table 1). None of the AAA patients took non-steroidal anti-inflammatory drugs (NSAIDs).

\subsection{Primary AAA-SMC cultures}

SMCs migrated from AAA explants after 10 days and reached confluence in one month. All the cultured AAA-SMCs had the same elongated, spindle-shaped morphology, and at confluence all cultures assumed a hill-and-valley pattern that was maintained throughout subcultures (Fig. 1A). Protein expression profiles determined by Western blot analysis (Fig. 1A) showed that all the cultures from patient groups expressed $\alpha$-SMA, vinculin and meta-vinculin, indicating the presence of differentiated SMCs. Fibronectin, a component of basement membrane, was also expressed indicating a proliferative and secretory SMC phenotype. RT-PCR analysis (Fig. 1B) indicated that mRNA expression of three genes appeared to be significantly down-regulated in SMCs from AAA patients: ACE (angiotensin converting enzyme), ADAMTS 5 and ADAMTS 8. The plots of individual mRNA expression for these three genes (normalized to house-keeping genes) shows the variability, with ADAMTS8 being the most suppressed.

\subsection{AAA-SMC proliferation and dimensions}

Despite similar morphologies within 2D cultures, the area and perimeter of AAA-SMCs within 2D cultures were significantly higher compared to their healthy counterparts (Fig. 2A; $p<0.01$ in both cases). The phenotype of AAA-SMCs was confirmed by staining for $\alpha$ actin in monolayer cultures, with more than $98 \%$ positive-stained cells. In general, AAA-SMC proliferation is significantly lower in 3D gels
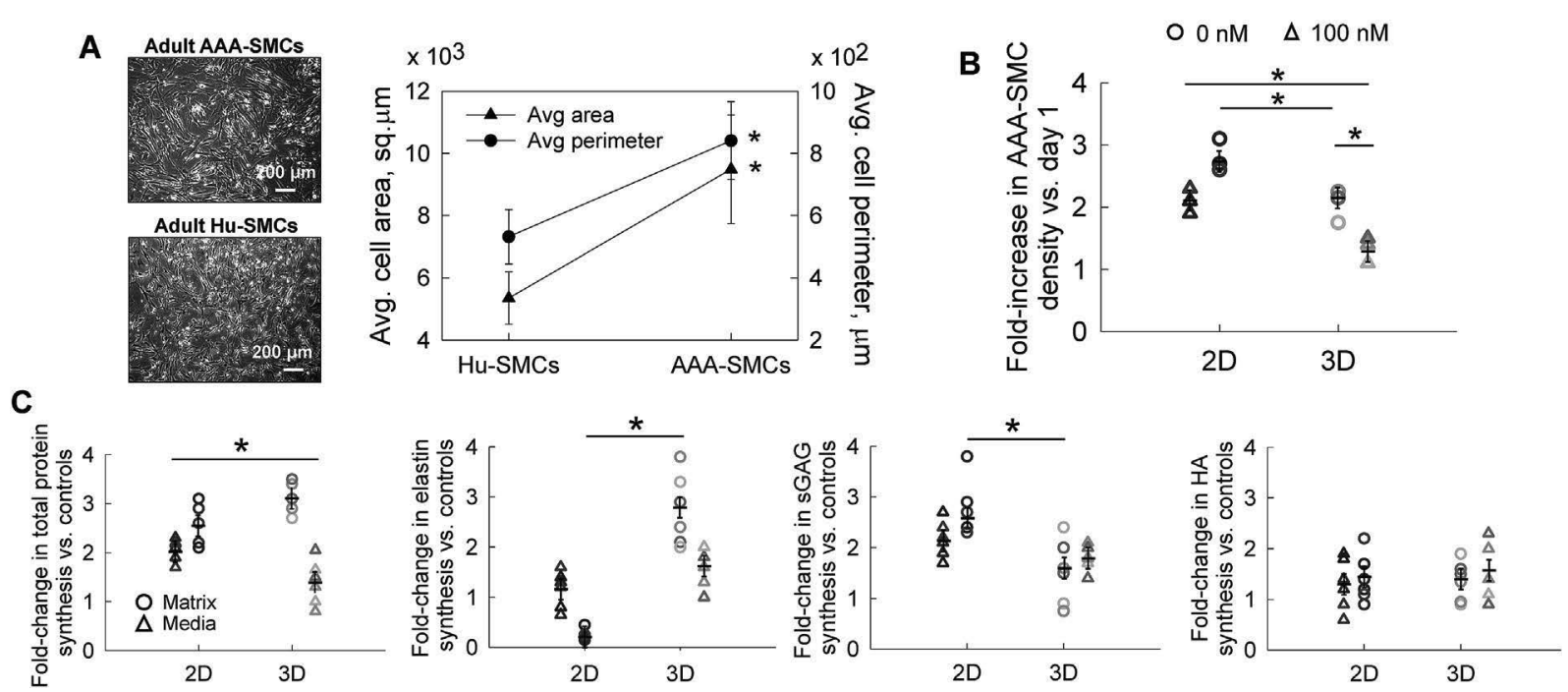

Fig. 2. (A) Representative phase-contrast images of adult healthy Hu-SMCs and AAA-SMCs cultured in this study. Significant differences in average cell area and perimeter were noted between the two cell types. Data shown represents mean \pm standard error in respective cases. Scale bar: $200 \mu \mathrm{m}$. (B) Fold increase in AAASMC density within 2D and 3D cultures, in the presence or absence of exogenous $100 \mathrm{nM}$ GSNO. Data shown represents mean \pm standard error of cell count after 21 days of culture, normalized to initial seeding density ( $n=3$ /condition). Cell densities were quantified using a fluorometric assay detailed earlier (C) Fold-changes (vs. GSNO-free controls). in total protein, elastin, sGAG, and HA, deposited within cell matrix or released into pooled media, by human AAA-SMCs, in 2D and 3D cultures, in the presence of $100 \mathrm{nM}$ GSNO. The total protein amounts were quantified using a BCA protein assay, elastin amounts using a Fastin Assay kit, sGAG amounts using a quantitative dye-binding sGAG assay kit, and HA amounts using a hyaluronan enzyme-linked immunosorbent quantitative assay. Quantitative results were obtained from cell matrix layers or pooled media after 21 days of culture and normalized to cell count within respective cases ( $\mathrm{n}=6 / \mathrm{condition)}$. Data were analyzed using one-way ANOVA followed by Tukey's HSD post-hoc testing, assuming unequal variance and differences deemed significant for $p<0.05$ between respective cases. Data shown represents mean \pm standard error in respective cases. The absolute amounts of each protein per cell were listed in Table 2 for baseline purposes. 
compared to their 2D counterparts (Fig. 2B). Addition of GSNO significantly suppressed SMC proliferation in 3D gels with a similar trend in $2 \mathrm{D}$ counterparts.

\subsection{Total protein synthesis}

Protein synthesis and release by AAA-SMCs has not been reported earlier. The total protein synthesized by AAA-SMCs, in 2D and 3D cultures, in the presence or absence of GSNO, was quantified as two separate components (Fig. 2C): protein deposited in the cell matrix (termed matrix here) and protein released into the pooled media (termed media here). A significant increase in the total protein deposited in cell matrix was observed with GSNO addition, in both 2D (2.58-fold) and 3D (3.1-fold) cultures. Total protein deposited in cell matrix within $3 \mathrm{D}$ cultures was significantly higher than that in $2 \mathrm{D}$ counterparts $(p<0.01)$, in the presence or absence of GSNO (16.4-fold and 19.8-fold, respectively). GSNO stimulated significant increase in the total protein released into the pooled spent media in 3D cultures $(p<0.01$ vs. 2D). The amounts of protein in pooled media were at least two-orders of magnitude higher than in matrix, in all conditions. This study established the baseline for the total protein amounts released and deposited by adult human AAA-SMCs in 2D and 3D cultures (actual amounts provided in Table 2), in the absence of any stimulation. However, less than $2 \%$ of total protein produced by AAA-SMCs was deposited as matrix within cell layers, suggesting that additional approaches are required to coacervate the protein into matrix form. Compared to healthy SMCs (Table 2; Supplementary Fig. 1), the total protein deposited within 3D cell layers or released into the spent media by AAA-SMCs was significantly higher (i.e., 2-fold and 19-fold, respectively) even in the absence of GSNO. GSNO stimulated a 7-fold increase in protein deposition within matrix layers and a 17-fold increase in protein release within pooled media by AAA-SMCs within 3D cultures, compared to Hu-SMCs (Supplementary Fig. 1).

\subsection{Elastin synthesis and deposition}

The total elastin synthesized by AAA-SMCs (Fig. 2C; Table 2) was quantified as that deposited in the cell layers (i.e., matrix elastin) or released into the pooled media (i.e., tropoelastin). The benefits of exogenous NO to elastin deposition in cell matrix appeared to be dependent on the substrate type. Compared to GSNO-free controls, significantly higher matrix elastin was deposited within 3D cultures with GSNO addition, although matrix elastin deposition decreased in 2D cultures. GSNO stimulated tropoelastin release only within 3D cultures, although the amounts were two-orders of magnitude higher than matrix elastin (Table 2).

The matrix elastin yields (ratio of matrix elastin to the total elastin produced) within 2D AAA-SMCs cultures were $0.4 \%$ and $0.08 \%$ with 0 and $100 \mathrm{nM}$ GSNO additive-cultures, respectively. However, 3D cultures modestly enhanced matrix elastin yields in the absence and presence of GSNO $(0.5 \%$ and $0.8 \%$, respectively). Thus, elastic matrix yields improved by 10 -fold in 3D cultures with the addition of GSNO, similar in trend to that observed for the total protein amounts.

\section{6. $s G A G$ and HA synthesis}

Adding GSNO to AAA-SMC cultures increased sGAG deposition in matrix layers (2.73- and 1.52- fold, respectively) and in pooled media (2.17- and 1.72- fold, respectively) within 2D and 3D cultures, per cell (Fig. 2C), although the amounts were two orders of magnitude higher in pooled media. Compared to $2 \mathrm{D}$ cultures, 3D cultures appeared to promote sGAG release into pooled media or deposition within cell layers, per cell (Table 2). sGAG yield (ratio of matrix sGAG to the total sGAG produced) within 2D AAA-SMC cultures indicated that matrix yields were $0.9 \%$ and $2.3 \%$ in controls and GSNO-additive cultures, respectively. However, 3D cultures did not show any enhancement of matrix sGAG yields, in the presence or absence of GSNO.

GSNO addition modestly increased HA deposition in cell matrix per cell in both 2D and 3D cultures (1.41- and 1.35- fold, respectively; Fig. 2C), with similar increases in HA release into the pooled media. When AAA-SMCs were cultured in 2D, the matrix HA yields (ratio of HA deposited in matrix to the total HA produced) were $1.6 \%$ and $1.7 \%$ within controls and GSNO additive-cultures, respectively. However, 3D cultures modestly increased matrix HA yields, in the presence or absence of GSNO ( $2 \%$ and $1.9 \%$, respectively).

\subsection{LOX enzyme activity}

While GSNO addition to 2D cultures did not induce a significant change in LOX activity within cell matrix, it induced a 2.55 -fold increase within 3D cultures (Fig. 3A; $p<0.01$ ). LOX activity in matrix within 3D cultures was significantly higher (2.2-fold; $p<0.01)$ than that in 2D cultures. LOX activity within pooled media from 3D cultures was 13.5-fold higher than that in 2D cultures in the absence of GSNO. Although adding GSNO to 3D cultures did not enhance LOX activity compared to controls, it significantly increased LOX activity within 2D cultures (14.9-fold; $p<0.01$ ).

\subsection{Desmosine crosslink density}

In healthy SMC cultures (Fig. 3B), desmosine levels were higher in $2 \mathrm{D}$ cultures than in 3D cultures $(p<0.01)$, and GSNO offered no additional benefit in both 2D and 3D cultures. However, in AAA-SMC cultures, desmosine levels were significantly higher in controls compared to GSNO-receiving cultures $(p<0.01)$, in both 2D and 3D. The basal levels of desmosine by AAA-SMCs remained unaltered in both 3D and 2D cultures. Finally, significant difference in desmosine levels were noted between healthy and AAA derived SMCs, in 3D cultures receiving

\section{Table 2}

Amounts of various proteins deposited in cell matrix or released into pooled media by healthy and AAA human aortic SMCs, per cell, over a 21-day period, within 2D or 3D cultures, in the presence or absence of $100 \mathrm{nM}$ GSNO. Data shown here represents the average of at least $\mathrm{n}=6 /$ case. Data for the healthy Hu-SMCs were from our previous publication [22].

\begin{tabular}{|c|c|c|c|c|c|c|c|c|c|c|c|c|c|}
\hline & & \multirow{2}{*}{$\begin{array}{l}\text { GSNO } \\
\text { dose } \\
(\mathrm{nM})\end{array}$} & \multirow{2}{*}{$\begin{array}{l}\text { Fold-increase in } \\
\text { proliferation, } \\
\text { compared to initial } \\
\text { seeding }\end{array}$} & \multicolumn{2}{|c|}{$\begin{array}{l}\text { Total protein } \\
\text { amounts }(\mu \mathrm{g})\end{array}$} & \multicolumn{2}{|l|}{ Elastin (ng) } & \multicolumn{2}{|l|}{ sGAGs (ng) } & \multicolumn{2}{|l|}{ HA (pg) } & \multicolumn{2}{|c|}{$\begin{array}{l}\text { LOX functional } \\
\text { activity (pM) }\end{array}$} \\
\hline & & & & $\begin{array}{l}\text { Cell } \\
\text { matrix }\end{array}$ & $\begin{array}{l}\text { Pooled } \\
\text { media }\end{array}$ & Cell matrix & $\begin{array}{l}\text { Pooled } \\
\text { media }\end{array}$ & Cell matrix & $\begin{array}{l}\text { Pooled } \\
\text { media }\end{array}$ & Cell matrix & $\begin{array}{l}\text { Pooled } \\
\text { media }\end{array}$ & $\begin{array}{l}\text { Cell } \\
\text { matrix }\end{array}$ & $\begin{array}{l}\text { Pooled } \\
\text { media }\end{array}$ \\
\hline \multirow[t]{2}{*}{ 3D cultures } & Healthy & 0 & 3.2 & 0.13 & 2.02 & ND & 7.06 & ND & 16.7 & 0.21 & 2.07 & 69 & 70 \\
\hline & Hu-SMCs & 100 & 2.03 & 0.12 & 3.12 & 1.31 & 29.9 & 0.49 & 7.08 & 0.5 & 3.2 & 370 & 1610 \\
\hline \multirow[t]{2}{*}{ 3D cultures } & Hu-AAA- & 0 & 2.05 & 0.27 & 37.9 & $<0.01$ & 0.17 & $<0.01$ & 0.036 & 0.57 & 27.6 & 0.1 & 2.76 \\
\hline & SMCs & 100 & 1.3 & 0.83 & 52.7 & $<0.01$ & 0.26 & $<0.01$ & 0.063 & 0.77 & 40.7 & 0.27 & 3.1 \\
\hline \multirow[t]{2}{*}{ 2D cultures } & Hu-AAA- & 0 & 2.8 & 0.016 & 16.8 & $<0.01$ & 0.15 & $<0.01$ & 0.015 & 0.36 & 22.8 & 0.02 & 0.18 \\
\hline & SMCs & 100 & 2.1 & 0.042 & 34.6 & $<0.01$ & 0.177 & $<0.01$ & 0.034 & 0.51 & 29.7 & 0.03 & 2.5 \\
\hline
\end{tabular}

ND: Not detectable within assay limits (i.e., $<1 \mathrm{pg} /$ cell amounts). 
A

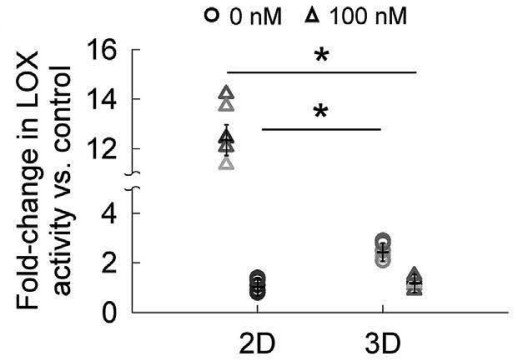

C
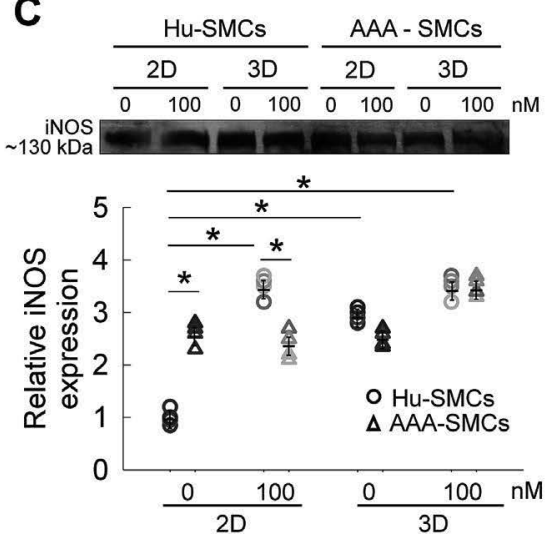

B

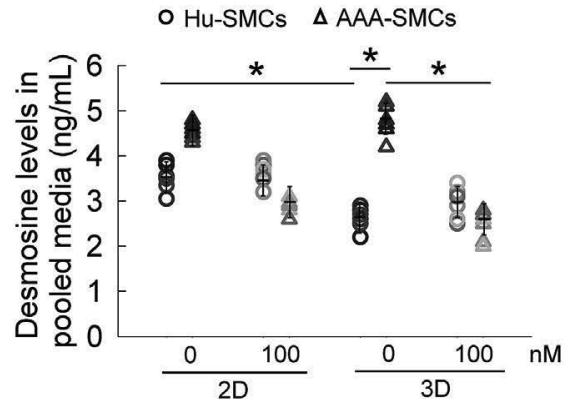

D

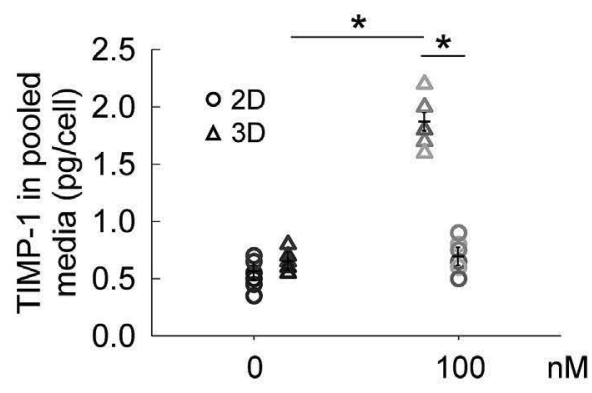

Fig. 3. (A) Fold-changes in LOX enzyme functional activity within cell matrix and pooled media of AAASMC cultures, quantified using an Amplex Red ${ }^{\circledast}$ fluorometric assay and quantified per cell. Cells were exposed to $100 \mathrm{nM}$ GSNO while controls received no GSNO. The absolute amounts of LOX functional activity per cell were listed in Table 2 for baseline purposes. (B) Desmosine levels in pooled media collected from 2D/3D Hu-SMCs or AAA-SMC cultures, over the 21-day period, in the presence or absence of GSNO. (C) iNOS protein expression was quantified from Western blot analysis. Representative bands are shown in the insert for comparison. iNOS protein expression by AAA-SMC cultures was normalized to that in 2D GSNO-free cultures of healthy SMCs. (D) Similarly, the release of TIMP-1 into pooled media was quantified using a TIMP-1 ELISA assay. GSNO (0 or $100 \mathrm{nM}$ ) was supplemented to AAA-SMC cultures in 2D or 3D cultures over 21 days. Data were analyzed using one-way ANOVA followed by Tukey's HSD post-hoc testing, assuming unequal variance and differences deemed significant for $p<0.05$. Data shown represent mean \pm standard error of quantified data and normalized to cell count within respective cases $(n=6$ per condition). (For interpretation of the references to color in this figure legend, the reader is referred to the Web version of this article.) no GSNO $(p<0.001)$

\section{9. iNOS expression and TIMP-1 release}

To test if exogenously-delivered NO influenced iNOS protein expression in AAA-SMC cultures, Western blot analysis was performed (Fig. 3C; bands in insert). The basal level of iNOS expression (2D control cultures) was significantly higher in AAA-SMCs compared to healthy counterparts $(p<0.05)$. It was found that GSNO addition to 2D cultures significantly promoted iNOS protein expression in healthy SMCs, but not in 3D cultures. Expression of iNOS by AAA-SMCs, on the other hand, was not modified by 2D or 3D cultures, and/or by GSNO addition.

No significant difference in TIMP-1 release was noted between 2D and 3D AAA-SMC cultures in controls (Fig. 3D). GSNO induced a 2.4fold increase in TIMP-1 release in 3D cultures. While the basal levels of TIMP-1 release was significantly lower in AAA-SMCs compared to adult healthy aortic SMCs in 3D cultures [22], the levels were similar in the presence of $100 \mathrm{nM}$ GSNO.

\subsection{Elastin, iNOS, LOX and fibrillin gene expression}

Elastin (ELN) gene expression in AAA-SMCs, both in 2D and 3D cultures, in the presence or absence of NO cues, was significantly lower compared to healthy SMCs (Fig. 4A). Such inhibited elastin gene expression levels in AAA-SMCs, compared to healthy SMCs, was in agreement with their respective protein expression levels of matrix elastin (Table 2). Similarly, within AAA-SMCs, elastin gene expression was significantly lower in $2 \mathrm{D}$ compared to that in $3 \mathrm{D}$, in agreement with their protein expression levels. Surprisingly, elastin gene expression was significantly lower after GSNO addition, in contrast to tropoelastin and matrix elastin produced by these cells (Fig. 2, C-D).

The iNOS gene expression of healthy and AAA-SMCs were similar in 2D and significantly increased in 3D cultures for AAA-SMCs (Fig. 4A). Adding GSNO had no significant effect on iNOS gene expression in AAA-SMC cultures, suggesting that AAA-SMCs are sensitive to signaling from exogenous NO cues, similar to their healthy counterparts. LOX gene expression was significantly higher (6-10 -fold in 2D and 3D cultures, respectively) in AAA-SMCs compared to healthy SMCs, although adding GSNO suppressed such elevated levels (Fig. 4A), in contrast with the LOX functional activity (Fig. 3A). The baseline levels of fibrillin-1 (FBN) gene in AAA-SMCs was significantly higher compared to their healthy counterparts, although such expression levels were significantly downregulated in 2D AAA-SMC cultures with GSNO addition (Fig. 4A).

\subsection{Release of cytokines, chemokines, MMPs, and TIMPs}

We quantified the baseline levels (2D - $0 \mathrm{nM}$ ) of cytokines, chemokines, MMPs and TIMPs released by healthy Hu-SMCs [Supplementary Fig. 2 (A: $<10 \mathrm{pg} / \mathrm{mL}$; C: $<250 \mathrm{pg} / \mathrm{mL}$ E: $<40 \mathrm{ng} / \mathrm{mL}$ )] and adult human AAA-SMCs [Supplementary Fig. 2 (B: $<10 \mathrm{pg} / \mathrm{mL}$; $\mathrm{D}:<250 \mathrm{pg} / \mathrm{mL} ; \mathrm{F}:<40 \mathrm{ng} / \mathrm{mL})$ ]. Their levels in 3D cultures, and in the presence of $100 \mathrm{nM}$ GSNO were also shown in respective panels. The analytes released by SMCs were plotted on a log-scale using a heatmap function (Fig. 4B). MMPs and TIMPs were the most highlyexpressed analytes compared to the cytokines (e.g., ILs). Benefits of GSNO delivery in healthy SMC cultures (both 2D and 3D), appeared marginal with no significant modulation in ILs and MMPs release but significant increase in TIMPs release. However, in 3D AAA-SMC cultures, GSNO suppressed the release of several analytes (e.g., IFN- $\gamma$, MMPs-8, 9, 12, IL-8) compared to all other conditions, and some analytes were undetectable in most conditions (IL-1 $\beta$ and TNF- $\alpha$ ). A tenfold increase in IL- 6 release by AAA-SMCs was found compared to healthy counterparts. Variations in analyte concentrations were observed across samples cultured in 2D vs. 3D environments; for instance, SMCs in 2D cultures had higher concentrations of MMP-2, MMP-12, TIMP-2, TIMP-3, and MCP-1, in both healthy and AAA-SMC cultures. In general, cytokine and MMPs production in both cell types appeared to be regulated by GSNO presence as well as culture substrate (2D vs. 3D).

\subsection{Immunofluorescence labeling of matrix proteins}

AAA-SMCs deposited detectable matrix elastin and fibulins- 4 and 5 within 2D cultures over the 21-day period within control cultures (Fig. 5). However, fibrillin-1, critical for matrix elastin coacervation 

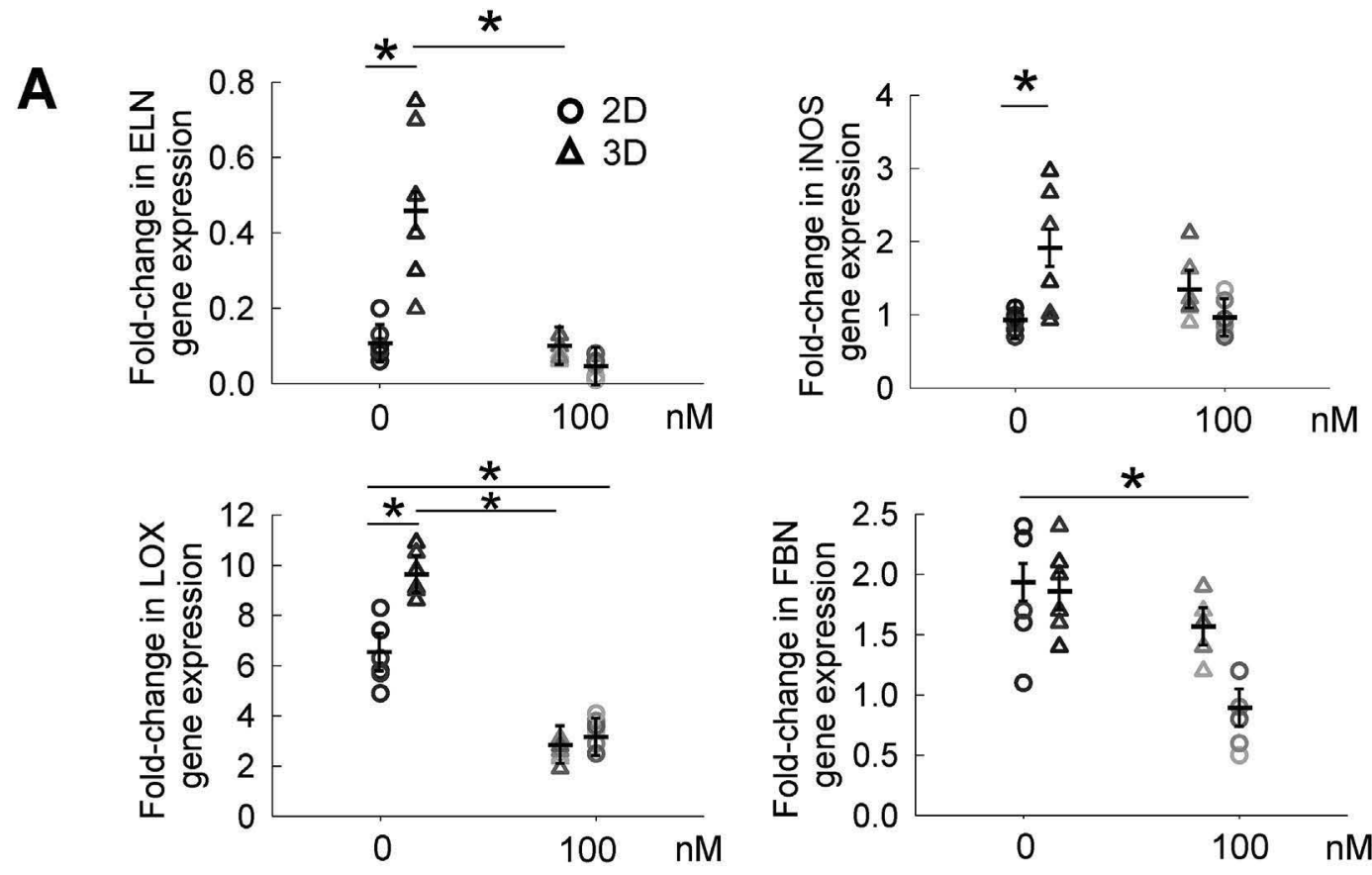

B
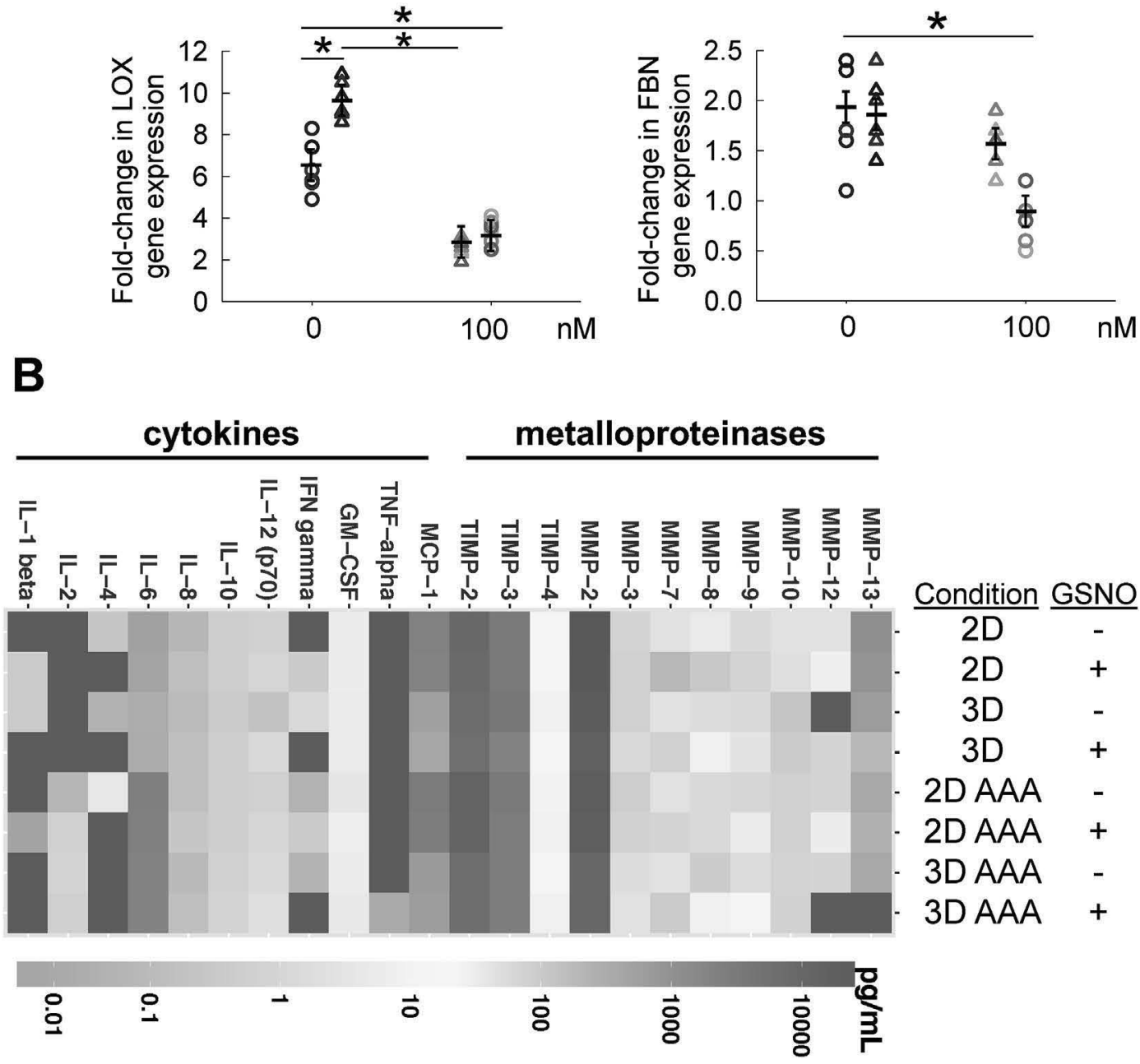

Fig. 4. (A) Lavik method ( $\triangle \Delta \mathrm{Ct}$ method) was used for relative quantification of the fold changes in ELN, iNOS, LOX, and FBN genes in AAA-SMCs, after addition of GSNO (0 or $100 \mathrm{nM}$ ) within 2D or 3D cultures for 21 days. The gene expressions obtained from qRT-PCR analysis within AAA-SMCs were first normalized to housekeeping gene (18S) expression within respective cultures, and further normalized to respective gene expressions within healthy Hu-SMCs cultured under similar conditions. Data were analyzed using one-way ANOVA followed by Tukey's HSD post-hoc testing, assuming unequal variance and differences deemed significant for $p<0.05$. Data shown represent mean \pm standard error $(n=6$ independent data points per condition). (B) Heat map showing the amounts of cytokines, chemokines, and MMPs/TIMPs released by healthy and AAA SMCs, in 2D and 3D cultures, in the presence or absence of $100 \mathrm{nM}$ GSNO, as measured using laser bead immunoassay.

and crosslinking, was undetectable around the periphery of most cells. GSNO promoted deposition of matrix elastin, fibrillin-1 and fibulins-4, 5 , compared to controls.

\subsection{Cell mechanics using AFM}

Single cell nanoindentation measurements were obtained to quantify the elastic modulus $(E)$ of live SMCs under different culture conditions (Fig. 6; Supplementary Fig. 3). Representative phase-contrast images (Supplementary Fig. 3), heat maps of elastic moduli (Fig. 6A), and force-indentation curves (Fig. 6B) were shown for healthy and AAA SMCs, in the presence or absence of GSNO. The average modulus of elasticity for AAA-SMCs $(17.41 \pm 0.34 \mathrm{kPa})$ was significantly higher (1.6-fold; $p<0.001)$ than for healthy SMCs $(10.42 \pm 0.29 \mathrm{kPa})$, indicating an increase of stiffness in AAA-SMCs (Fig. 6C). Upon exposure to GSNO, the average modulus of elasticity for healthy SMCs decreased by 1.3 -fold (healthy SMCs: $8.67 \pm 0.25 \mathrm{kPa}, p<0.05$; AAA-SMCs: $13.03 \pm 0.35 \mathrm{kPa}, p<0.05)$. Cell roughness measurements showed 

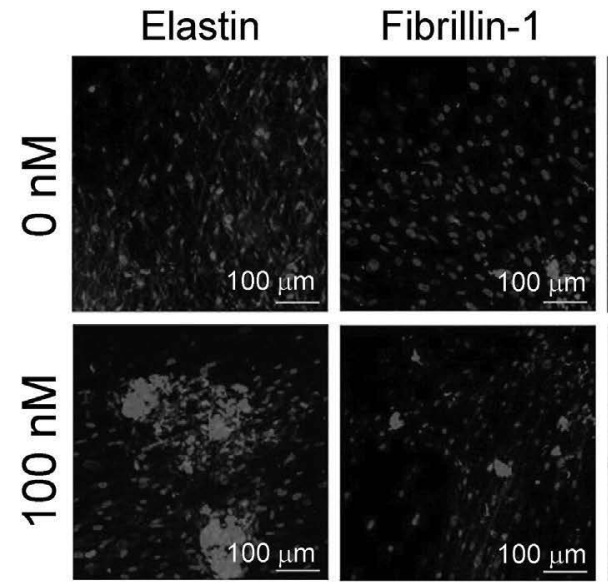

Fibulin-4

Fibulin-5
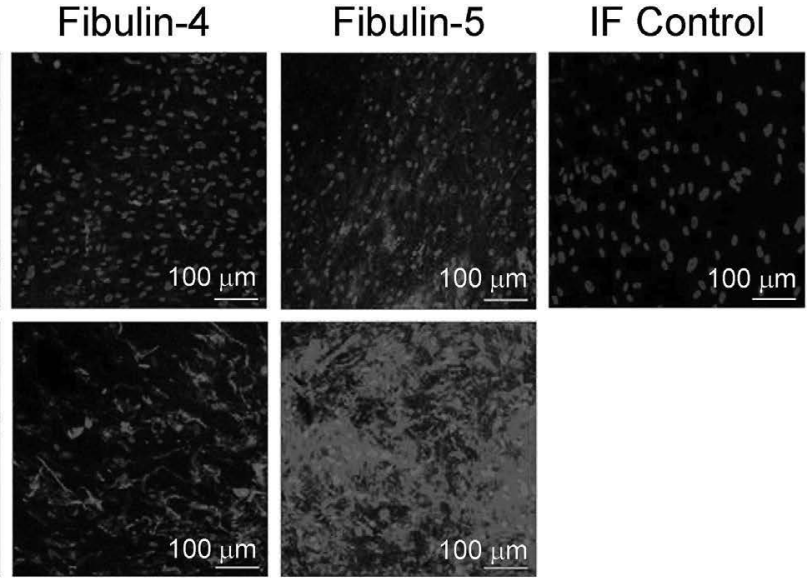

Fig. 5. Representative immunofluorescence images of AAA-SMC layers in 2D cultures, with or without GSNO, stained with respective primary antibodies for elastin, fibrillin-1, fibulin-4 and fibulin-5, and counterstained with DAPI for cell nuclei. A significant increase in the expression of all the proteins was noted in the presence of $100 \mathrm{nM}$ GSNO. Scale bar: $100 \mu \mathrm{m}$. For each treatment condition, at least three independent cell layers were imaged, with six random regions captured in each cell layer.

that AAA-SMCs were smoother than Hu-SMCs (Fig. 6D). GSNO addition significantly increased cell surface roughness in Hu-SMCs $(p<0.01)$, and nearly doubled the roughness in AAA-SMCs, compared to respective controls. In the presence of GSNO, Hu-SMCs and AAA-SMCs had no significant differences in their surface roughness.

\section{Discussion}

In this study, we compared the differences and behaviors of human aneurysmal SMCs and their healthy counterparts regarding cell proliferation, phenotypic and gene expression, transcriptomic analyses, immunofluorescence imaging, biomechanical characteristics, and their response to exogenously-delivered NO cues, as a therapeutic AAA
A

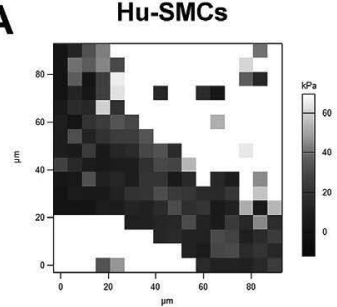

Hu-SMCs + GSNO

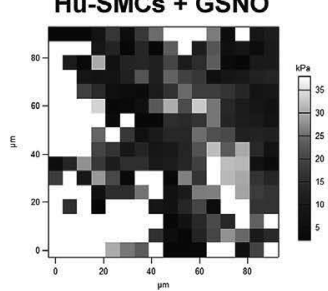

D
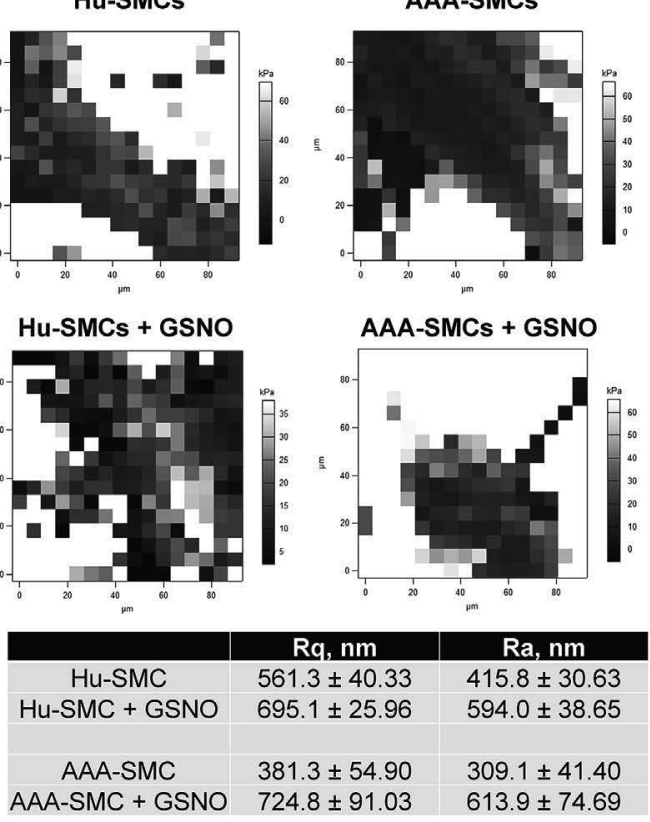

AAA-SMCs + GSNO

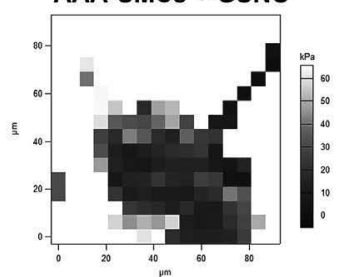

treatment.

Prior studies reported that adult human AAA-SMCs proliferated 4fold over 16 days [37], 5 -fold over 21 days [38], or doubled every 5.3 days [39] in 2D cultures, which is significantly higher than that noted in our study. AAA-SMCs proliferated significantly slower compared to their healthy counterparts (Table 2), possibly due to diseased nature and/or minor differences in the phenotype between donors. AAA-SMCs synthesized and deposited more proteins in 3D cultures compared to the healthy aortic SMCs. Our results highlight the increased ability of aortic SMCs to proliferate, migrate and deposit ECM under diseased vascular conditions [40].

Despite the prominent role of elastin in aortic tissue homeostasis maintenance, quantification of elastin synthesized by human AAA-

B

C
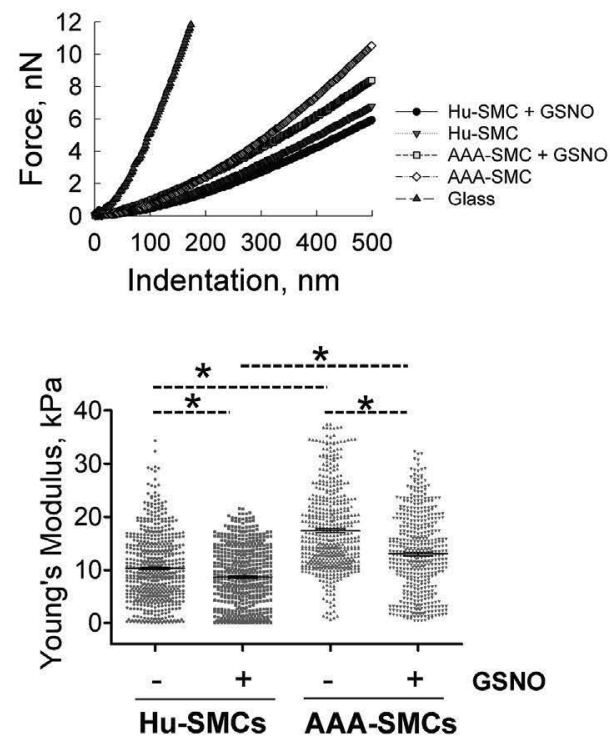

Fig. 6. (A) Representative force maps of healthy Hu-SMCs (left panels) and AAA-SMCs (right panels) treated with $100 \mathrm{nM}$ GSNO (lower panels). Background from Petri dish was subtracted in these images. Representative force-indentation curves (B) and average Young's Modulus (C) of these cells cultured on 2D substrates, in the presence or absence of $100 \mathrm{nM}$ GSNO. The black dotted lines in (C) represent the arithmetic averages in respective cases, i.e., $10.33 \pm 0.2624 \mathrm{KPa}$ for untreated Hu-SMCs $(n=605), 8.674 \pm 0.2491 \mathrm{KPa}$ for Hu-SMCs receiving GSNO $(n=550), 17.41 \pm 0.3405 \mathrm{KPa}$ for untreated AAA-SMCs ( $n=524)$, and $13.03 \pm 0.3498 \mathrm{KPa}$ for AAA-SMCs receiving GSNO $(n=469)$. (D) The surface roughness parameters Rq and Ra in Hu-SMCs and AAA-SMCs, in the presence of absence of GSNO. Data were analyzed using one-way ANOVA followed by Tukey's HSD post-hoc testing, assuming unequal variance and differences deemed significant for $p<0.05$. Data shown represent mean \pm standard error $(n=6$ independent data points per condition). 
SMCs has received less attention. Tropoelastin, the precursor molecule to elastin, is encoded by a single copy of ELN gene in SMCs and fibroblasts. Tropoelastin amounts in human AAA extracts was reported to be 5-fold higher than that within normal human aortic tissues [21]. Our current study suggests that healthy Hu-SMCs produce higher amounts of tropoelastin and matrix elastin compared to their AAA counterparts. Adult human AAA-SMCs were shown to release $\sim 10 \mathrm{pg}$ of tropoelastin and deposited $0.02 \mathrm{pg}$ of matrix elastin per cell in $2 \mathrm{D}$ cultures, in the absence of any external cues [38]. These amounts were significantly lower than what we observed, despite similarities in donor age, disease phenotype and culture duration. Numerous studies have shown that the significant changes in elastin protein within AAA ECM might be due to a related change in the gene expression levels [41,42]. Similar to our observations here, a down-regulation in elastin gene in AAA tissues was reported in a porcine model of induced AAA, compared to healthy counterparts [43].

Adult human AAA-SMCs in 2D cultures release significantly higher levels of GAGs (e.g., versican, perlecan, heparan sulfate, biglycans) compared to healthy aortic SMCs [44]. Although the various types of sGAGs released by AAA-SMCs were not individually identified in our study, we quantified the absolute amount of overall GAGs production by AAA-SMCs and found multifold increases in HA release into pooled media and deposition into matrix within AAA-SMC cultures. The combined amount of elastin and GAGs, in both pooled media and cell matrix, constitute a small fraction of the total matrix, suggesting that in addition to elastin and GAGs, numerous other proteins such as collagens and proteoglycans, might have been released and deposited by AAA-SMCs within 2D and 3D cultures.

Such deposited elastin precursors are crosslinked in the presence of LOX enzyme, resulting in the formation of desmosine and isodesmosine linkages between lysine molecules on elastin molecules [45]. Thus, the final crosslinked elastic fibers typically have a microfibril sheath surrounding the core elastin molecules. Additional stimulation such as alternate scaffold design, elastogenic scaffold material, exogenous LOX, and mechanical stimuli might be necessary to coax the AAA-SMCs to enhance matrix elastin yields. Although it is interesting to note that AAA-SMCs produce LOX which is functionally active, the levels of LOX functional activity within AAA-SMC cultures are at least two orders of magnitude lower than that quantified within healthy SMC cultures (Table 2), per cell. This difference might be due to the phenotypic differences between healthy versus AAA cells. Studies have shown that in addition to regulating vascular SMC phenotype (proliferation, migration), LOX also plays a primary role in ECM crosslinking such as collagens, elastin and GAGs [46]. However, under diseased conditions such as aneurysms, characterized by remarkable degradation and ECM remodeling, LOX activity and expression is significantly reduced. While it is not clear whether reduction in LOX levels contribute to the onset of the pathogenesis of these diseases or vice versa, numerous AAA-induced animal models demonstrate strong correlation between LOX expression reduction and disease progression [47]. Desmosine levels were detectable even in AAA-SMC cultures attesting to their ability to release components critical for crosslinked elastin deposition, although GSNO presence did not further stimulate these levels. Prior studies have shown that desmosine levels in healthy human arterial tissues were at least 9-fold higher than that in AAA segments [21], and reduced desmosine levels were reported in dissecting AAAs [48], suggesting the reason for diminished levels of mature, crosslinked elastin in AAA tissues.

Although vascular NO plays an important role in regulating SMC functions such as vasodilation, survival, gene regulation, and quiescence phenotype, the harmful role of higher NO levels in the pathogenesis of aneurysms has been documented [49-51]. Specifically, iNOSstimulated NO release from vascular SMCs was shown to participate in elastin degradation and disruption of vascular ECM [52]. Our observations agree with previous reports that experimental or human AAA-derived tissues had higher iNOS levels compared to control tissues where iNOS could barely be detected [50,53]. TIMPs-1, 2 regulate ECM remodeling in vasculature by controlling the levels and activity of MMPs. In agreement with our results, no significant differences in TIMP-1 levels (Western blotting) within tissue sections isolated from AAA patients and their healthy counterparts were observed [54].

Localized inflammation contributes to weakening of aorta due to imbalances in ECM levels [55]. This has prompted research focused on regulating the production of inflammatory molecules as a therapeutic intervention in lieu of invasive surgical approaches [56]. The concentrations noted for MMPs/TIMPs in our study broadly falls in the range reported by others and our previous work [22,37]. Given the apparent differences in cytokine production between AAA and healthy SMCs, our data supports the prevailing notion that cytokine stimulation via paracrine or autocrine signaling may in turn modulate MMP activation [57]. Although it has been suggested that NO can influence the production of some cytokines and chemokines [58], we did not see any differences. In summary, our data is consistent with the inflammatory patterns seen in AAA subjects and suggests that NO presence modulates MMP production in a potentially therapeutic manner.

In addition to alterations in composition and functionality of vascular endothelium and ECM, changes in cell stiffness might also contribute to vascular tissue stiffness in AAAs, atherosclerosis and hypertensive conditions. The stiffness $(\sim 30 \mathrm{kPa})$ of hypertensive aortaderived SMCs, as measured by AFM nano-indentation, was reported to be two-fold higher than that of normal aorta $(\sim 14 \mathrm{kPa})$, and such stiffness was mediated by cytoskeletal proteins [59]. Vascular SMCs from old male monkeys exhibited much higher stiffness than their younger counterparts (42 kPa $v s .13 \mathrm{kPa}$ ) [60]. The elastic modulus of primary adult male rat arteriole-derived SMCs $(\sim 13.5 \mathrm{kPa})$ decreased by $38 \%$ upon exposure to NO donor PANOate, most likely due to remodeling of actin cytoskeleton network [61]. Marfan vascular SMCs were two-fold stiffer than healthy human SMCs, as measured by AFM, most likely due to higher focal adhesion kinase protein and actin stress fibers expression [62]. Here, we report for the first time on the stiffness and surface roughness of human aortic SMCs from healthy and AAA tissues, using AFM nano-indentation of live cells. Our results show that (i) elastic modulus of healthy Hu-SMCs is lower than that in rats and monkeys; (ii) human AAA-SMCs have higher stiffness and lower surface roughness compared to healthy Hu-SMCs; and (iii) human AAA-SMCs had significantly lower stiffness compared to hypertensive rat SMCs or aged monkey SMCs.

Our results support our hypothesis that AAA-SMCs do respond to exogenous NO cues to modulate cell proliferation and protein synthesis in $3 \mathrm{D}$ cultures. We have recently shown that healthy Hu-SMCs produced significantly higher amounts of tropoelastin and matrix elastin in the presence of $100 \mathrm{nM} \mathrm{GSNO}$, and that an optimal $100 \mathrm{nM}$ GSNO promoted sGAG deposition in matrix layers but not their release into the pooled media, within 3D Hu-SMC cultures [22]. Our results along with pertinent literature suggest that exogenously-delivered NO donors (e.g., GNSO) release NO and other reaction products which stimulate iNOS expression and endogenous NO release in healthy or aneurysmal SMC cultures (Fig. 7). Such endogenous NO upregulates cGMP and thereby protein kinase G (PKG) activation in SMCs $[23,63]$, which in turn induce cell relaxation (i.e., low stiffness) [64], suppress proliferation [65], and stimulate elastic matrix synthesis [23]. This cascade of events might contribute to enhanced SMC phenotype leading to improvement in vascular tone and compliance in vivo, desired from a therapeutic standpoint.

An increase in LOX functional activity within 3D AAA-SMC cultures in GSNO presence (Fig. 3), and significantly higher deposition of fibrillar forms of elastic matrix-associated proteins such as fibrillin-1, and fibulins- 4, 5 (Fig. 5), is highly encouraging for therapeutic tissue engineering and in situ elastin regeneration. Increased levels of LOX functional activity by exogenous GSNO might partially explain the increases in matrix elastin, GAGs, and tropoelastin in 3D AAA-SMC cultures. Similar increases in tropoelastin but not desmosine levels by 


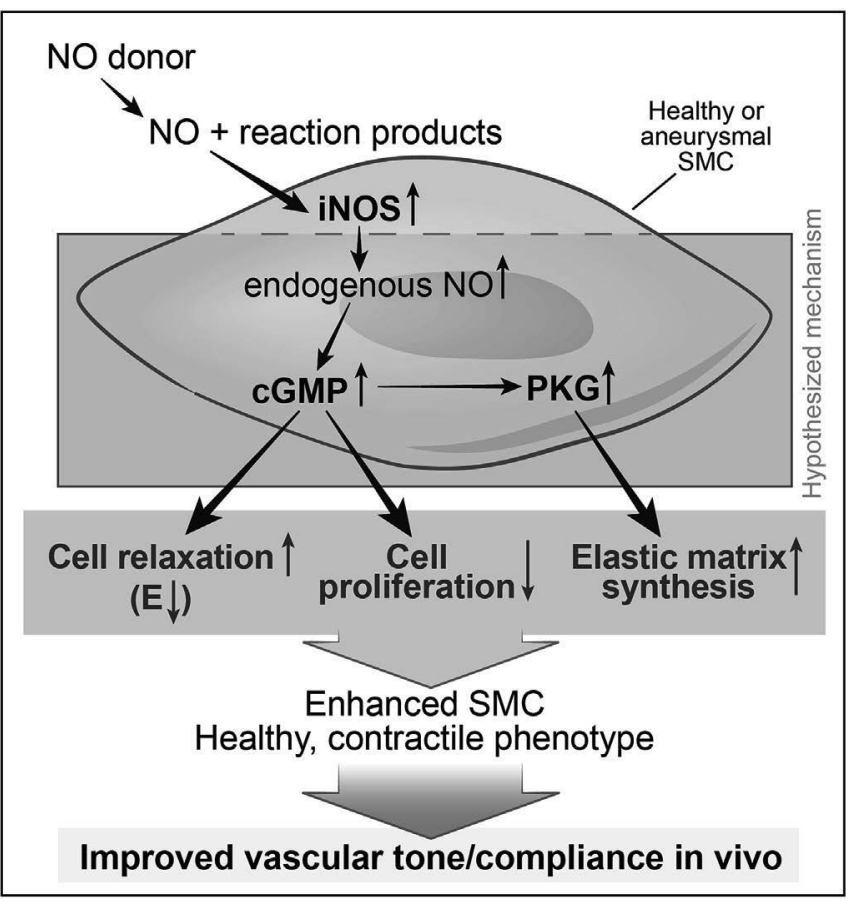

Fig. 7. Schematic of the hypothesized mechanism by which exogenously-delivered nitric oxide (NO) donors such as GSNO ultimately influence smooth muscle cell (SMC) phenotype and genotype in both healthy and aneurysmal aorta. Sequentially, the NO released by donors upregulates iNOS gene expression and manifests as endogenous NO release, which in turn increases cyclic guanosine monophosphate (cGMP) and protein kinase G (PKG) expression, thereby enhancing cell relaxation, suppressing proliferation, and promoting elastic matrix synthesis. These together contribute to heightened contractile SMC phenotype and improved vasculature tone and compliance in vivo. Here $E$ refers to elastic modulus, an estimate of the cell stiffness.

human and rat AA-SMCs were reported by others [21,66]. Higher amounts of GSNO ( $>100 \mathrm{nM}$ ) could possibly elevate desmosine levels in 3D AAA-SMC cultures, which further enhances elastin crosslinking and maturation.

The increased levels of TIMP-1 in AAA-SMC 3D cultures in the presence of GSNO is encouraging in modulating further degradation of vascular ECM by MMPs under diseased conditions. GSNO induced significant increases in matrix elastin deposition in 3D cultures and reduced MMP-9 concentration by over $50 \%$ in all cases (2D/3D AAA-SMC cultures), compared to healthy controls. Given that the upregulation of MMP-9 is linked to the degradation of the aortic ECM, our results are encouraging as they suggest a therapeutic benefit in the supplementation of exogenous NO [67]. Similar trend was noted for MMP-2 in nearly all cases (excluding 3D AAA-SMC cultures). Similar patterns in MMP downregulation were observed in eNOS gene-transfected cells [68], and it has been suggested this pathway is regulated by guanylylcyclase dependent and independent pathways [69]. NO appeared to have little effect on TIMPs release across most cases. In agreement with literature, we noticed significantly higher IL- 6 levels by AAA-SMCs. And finally, delivering exogenous NO significantly lessened AAA-SMC stiffness and enhanced surface roughness, possibly via modulation of cytoskeletal and surface proteins, respectively. We postulate that changes in stiffness beginning at single cell level might contribute to changes in vascular tone and functionality of aneurysmal vessels and delivering GSNO could be explored as a therapeutic strategy. Taken together, results confirm our hypothesis that exogenous NO delivery to AAA-SMCs might help to promote protein synthesis, release and deposition within 3D cultures, in addition to regulating their proliferation.

In conclusion, we established the baseline differences between adult human AAA and healthy SMCs, in terms of their size, proliferation, matrix and associated proteins synthesis and deposition, release of cytokines/chemokines/MMPs/TIMPs, changes in gene expression of elastogenic markers, and stiffness and roughness. We demonstrated that exogenously delivering NO via GSNO offers an effective strategy to enhance AAA-SMC functionality. Our current studies are geared towards validating these phenotypic, genetic expression, and biomechanical outcomes in multiple patient-derived AAA and healthy SMCs. Nevertheless, these results help in designing tissue engineering and regenerative medicine-based approaches, consisting of 3D scaffolds and localized drug delivery options, specifically for in situ elastin regeneration and remodeling to arrest aneurysm progression.

\section{Declarations of interest}

None.

\section{Sources of Funding}

This work was partially supported by the Cleveland State University Cellular and Molecular Medicine Fellowships and the Dissertation Research Awards to J.J. and K.F., the Choose Ohio First Scholarship to P.S., the National Science Foundation (CBET, Award \# 1337859) to C.K., the European Fighting Aneurysmal disease grant (Health-F22008-200647) to F.P., and the National Institutes of Health (HL132856 and HL119810-05; Project Number: NCAI-17-7-APP-CCF-Ramamurthi) to A.R. The funding sources have no role in study design; in the collection, analysis and interpretation of data; in the writing of the report; and in the decision to submit the article for publication.

\section{Acknowledgements}

The authors acknowledge help from Dr. Ali Borazjani (Cleveland Clinic) with RT-PCR analysis, Sarah Carney with confocal imaging, and Ms. Arsela Gishto with biochemical assays.

\section{Appendix A. Supplementary data}

Supplementary data to this article can be found online at https:// doi.org/10.1016/j.yexcr.2019.111589.

\section{References}

[1] S. Aggarwal, A. Qamar, V. Sharma, A. Sharma, Abdominal aortic aneurysm: a comprehensive review, Exp. Clin. Cardiol. 16 (2011) 11.

[2] M.A. Dale, M.K. Ruhlman, B.T. Baxter, Inflammatory cell phenotypes in AAAs: their role and potential as targets for therapy, Arterioscler. Thromb. Vasc. Biol. 35 (2015) 1746-1755.

[3] C.G. Solomon, K.C. Kent, Abdominal aortic aneurysms, N. Engl. J. Med. 371 (2014) 2101-2108.

[4] B.S. Brooke, S.K. Karnik, D.Y. Li, Extracellular matrix in vascular morphogenesis and disease: structure versus signal, Trends Cell Biol. 13 (2003) 51-56.

[5] P. Lacolley, V. Regnault, A. Nicoletti, Z. Li, J.-B. Michel, The vascular smooth muscle cell in arterial pathology: a cell that can take on multiple roles, Cardiovasc Res. 95 (2012) 194-204.

[6] H. Kuivaniemi, E.J. Ryer, J.R. Elmore, G. Tromp, Understanding the pathogenesis of abdominal aortic aneurysms, Expert Rev. Cardiovasc Ther. 13 (2015) 975-987.

[7] A.J. Bank, H. Wang, J.E. Holte, K. Mullen, R. Shammas, S.H. Kubo, Contribution of collagen, elastin, and smooth muscle to in vivo human brachial artery wall stress and elastic modulus, Circulation 94 (1996) 3263-3270.

[8] J.E. Wagenseil, R.P. Mecham, Vascular extracellular matrix and arterial mechanics, Physiol. Rev. 89 (2009) 957-989.

[9] B. Sivaraman, C.A. Bashur, A. Ramamurthi, Advances in biomimetic regeneration of elastic matrix structures, Drug Deliv. Transl. Res. 2 (2012) 323-350.

[10] B.S. Brooke, A. Bayes-Genis, D.Y. Li, New insights into elastin and vascular disease, Trends Cardiovasc. Med. 13 (2003) 176-181.

[11] E. Arteaga-Solis, B. Gayraud, F. Ramirez, Elastic and collagenous networks in vascular diseases, Cell Struct. Funct. 25 (2000) 69.

[12] R.A. Fraga-Silva, B. Trachet, N. Stergiopulos, Emerging pharmacological treatments to prevent abdominal aortic aneurysm growth and rupture, Curr. Pharmaceut. Des. 21 (2015) 4000-4006.

[13] F.M. Davis, D.L. Rateri, A. Daugherty, Abdominal aortic aneurysm: novel mechanisms and therapies, Curr. Opin. Cardiol. 30 (2015) 566-573.

[14] N. Patelis, D. Moris, G. Karaolanis, S. Georgopoulos, Endovascular vs. Open repair for ruptured abdominal aortic aneurysm, Med. Sci. Mon. Basic Res. 22 (2016) 
34-44.

[15] M.D. Tilson, Decline of the atherogenic theory of the etiology of the abdominal aortic aneurysm and rise of the autoimmune hypothesis, J. Vasc. Surg. 64 (2016) 1523-1525.

[16] C.A. Bashur, L. Venkataraman, A. Ramamurthi, Tissue engineering and regenerative strategies to replicate biocomplexity of vascular elastic matrix assembly, Tissue Eng. Part B 18 (2012) 203-217.

[17] C.A. Bashur, R.R. Rao, A. Ramamurthi, Perspectives on stem cell-based elastic matrix regenerative therapies for abdominal aortic aneurysms, Stem Cells Transl. Med. 2 (2013) 401-408.

[18] C.J. O'Callaghan, B. Williams, Mechanical strain-induced extracellular matrix production by human vascular smooth muscle cells - role of TGF-beta(1), Hypertension 36 (2000) 319-324.

[19] C. Erikstrup, L.M. Pedersen, L. Heickendorff, T. Ledet, L.M. Rasmussen, Production of hyaluronan and chondroitin sulphate proteoglycans from human arterial smooth muscle - the effect of glucose, insulin, IGF-1 or growth hormone, Eur. J. Endocrinol. 145 (2001) 193-198.

[20] J.E. Figueroa, J. Oubre, P. Vijayagopal, Modulation of vascular smooth muscle cells proteoglycan synthesis by the extracellular matrix, J. Cell. Physiol. 198 (2004) 302-309.

[21] A. Krettek, G.K. Sukhova, P. Libby, Elastogenesis in human arterial disease: a role for macrophages in disordered elastin synthesis, Arterioscler. Thromb. Vasc. Biol. 23 (2003) 582-587.

[22] P. Simmers, A. Gishto, N. Vyavahare, C.R. Kothapalli, Nitric oxide stimulates matrix synthesis and deposition by adult human aortic smooth muscle cells within threedimensional cocultures, Tissue Eng. A 21 (2015) 1455-1470.

[23] H. Sugitani, H. Wachi, S. Tajima, Y. Seyama, Nitric oxide stimulates elastin expression in chick aortic smooth muscle cells, Biol. Pharm. Bull. 24 (2001) 461-464.

[24] W.K. Alderton, C.E. Cooper, R.G. Knowles, Nitric oxide synthases: structure, function and inhibition, Biochem. J. 357 (2001) 593-615.

[25] S.M. Black, R.S. Heidersbach, D.M. McMullan, J.M. Bekker, M.J. Johengen, J.R. Fineman, Inhaled nitric oxide inhibits NOS activity in lambs: potential mechanism for rebound pulmonary hypertension, Am. J. Physiol. 277 (1999) H1849-H1856.

[26] A.M. Sheehy, M.A. Burson, S.M. Black, Nitric oxide exposure inhibits endothelial NOS activity but not gene expression: a role for superoxide, Am. J. Physiol. 274 (1998) L833-L841.

[27] J.S. Stamler, S. Lamas, F.C. Fang, Nitrosylation. the prototypic redox-based sig naling mechanism, Cell 106 (2001) 675-683.

[28] U.C. Garg, A. Hassid, Nitric oxide generating vasodilators and 8-bromo-cyclic guanosine monophosphate inhibit mitogenesis and proliferation of cultured rat vascular SMCs, J. Clin. Investig. 83 (1989) 1774-1776.

[29] I.J. Kullo, R.S. Schwartz, V.J. Pompili, M. Tsutsui, S. Milstien, L.A. Fitzpatrick, Z.S. Katusic, T. O'Brien, Expression and function of recombinant endothelial NO synthase in coronary artery smooth muscle cells, Arterioscler. Thromb. Vasc. Biol. 17 (1997) 2405-2412.

[30] J. Sato, K. Nair, J. Hiddinga, N. Eberhardt, L.A. Fitzpartrick, Z.S. Katusic, T. O'Brien, eNOS gene transfer to vascular smooth muscle cells inhibits cell proliferation via upregulation of p27 and p21 and not apoptosis, Cardiovasc. Res. 47 (2000) 697-706.

[31] Y. Mitani, S.H.E. Zaidi, P. Dufourcq, K. Thompson, M. Rabinovitch, Nitric oxide reduces vascular smooth muscle cell elastase activity through cGMP-mediated suppression of ERK phosphorylation and AML1B nuclear partitioning, FASEB J. 14 (2000) 805-814.

[32] E. Lopez, O. Boucherat, M.L. Franco-Montoya, J.R. Bourbon, C. Delacourt, P.H. Jarreau, Nitric oxide donor restores lung growth factor and receptor expression in hyperoxia-exposed rat pups, Am. J. Respir. Cell Mol. Biol. 34 (2006) 738-745.

[33] N. Lamblin, P. Ratajczak, D. Hot, E. Dubois, M. Chwastyniak, O. Beseme, H. Drobecq, Y. Lemoine, M. Koussa, P. Amouyel, F. Pinet, Profile of macrophages in human abdominal aortic aneurysms: a transcriptomic, proteomic, and antibody protein array study, J. Proteome Res. 9 (2010) 3720-3729.

[34] B. Hama, G. Mahajan, C.R. Kothapalli, Characterizing viscoelasticity of unhydrolyzed chicken sternal cartilage extract suspensions: towards development of injectable therapeutics formulations, J. Mech. Behav. Biomed. Mater. 72 (2017) 90-101.

[35] Q.S. Li, G.Y.H. Lee, C.N. Ong, C.T. Lim, AFM indentation study of breast cancer cells, Biochem. Biophys. Res. Commun. 374 (2008) 609-613.

[36] K.S. Kim, C.H. Cho, E.K. Park, M.H. Jung, K.S. Yoon, H.K. Park, AFM-detected apoptotic changes in morphology and biophysical property caused by paclitaxel in Ishikawa and HeLa cells, PLoS One 7 (2012) e30066.

[37] M. Crowther, S. Goodall, J.L. Jones, P.R. Bell, M.M. Thompson, Increased matrix metalloproteinase 2 expression in vascular smooth muscle cells cultured from abdominal aortic aneurysms, J. Vasc. Surg. 32 (2000) 575-583.

[38] C. Gacchina, T. Brothers, A. Ramamurthi, Evaluating smooth muscle cells from $\mathrm{CaCl} 2$-induced rat aortal expansions as a surrogate culture model for study of elastogenic induction of human aneurysmal cells, Tissue Eng. A 17 (2011) 1945-1958.

[39] M.I. Patel, P. Ghosh, J. Melrose, M. Appleberg, Smooth muscle cell migration and proliferation is enhanced in abdominal aortic aneurysms, Aust. N. Z. J. Surg. 66 (1996) 305-308.

[40] G.K. Owens, M.S. Kumar, B.R. Wamhoff, Molecular regulation of vascular smooth muscle cell differentiation in development and disease, Physiol. Rev. 84 (2004) 767-801.

[41] C.L. Mesh, B.T. Baxter, W.H. Pearce, R.L. Chisholm, G.S. McGee, J.S. Yao, Collagen and elastin gene expression in aortic aneurysms, Surgery 112 (1992) 256-261.

[42] M. Wassef, B.T. Baxter, R.L. Chisholm, R.L. Dalman, M.F. Fillinger, J. Heinecke, J.D. Humphrey, a. et, Pathogenesis of abdominal aortic aneurysms: a multidisciplinary research program supported by the National Heart, Lung, and Blood Institute, J. Vasc. Surg. 34 (2001) 730-738.

[43] M. Sadek, R.L. Hynecek, S. Goldenberg, K.C. Kent, M.L. Marin, P.L. Faries, Gene expression analysis of a porcine native abdominal aortic aneurysm model, Surgery 144 (2008) 252-258.

[44] J. Melrose, J. Whitelock, Q. Xu, P. Ghosh, Pathogenesis of abdominal aortic aneurysms: possible role of differential production of proteoglycans by smooth muscle cells, J. Vasc. Surg. 28 (1998) 676-686.

[45] C.E. Schmelzer, Assembly and Properties of Elastic Fibers, Elastic Fiber Matrices: Biomimetic Approaches to Regeneration and Repair, CRC Press, 2016, pp. 1-30.

[46] J.M. Maki, R. Sormunen, S. Lippo, R. Kaarteenaho-Wiik, R. Soininen, J. Myllyharju, Lysyl oxidase is essential for normal development and function of the respiratory system and for the integrity of elastic and collagen fibers in various tissues, Am. J. Pathol. 167 (2005) 927-936.

[47] C. Rodríguez, J. Martínez-González, B. Raposo, J.F. Alcudia, A. Guadall, L. Badimon, Regulation of lysyl oxidase in vascular cells: lysyl oxidase as a new player in cardiovascular diseases, Cardiovasc. Res. 79 (2008) 7-13.

[48] M. Watanabe, T. Sawai, Alteration of cross-linking amino acids of elastin in human aorta in association with dissecting aneurysm: analysis using high performance liquid chromatography, Tohoku J. Exp. Med. 187 (1999) 291-303.

[49] J.M. Johanning, P.J. Armstrong, D.P. Franklin, D.C. Han, D.J. Caney, J.R. Elmore, Nitric oxide in experimental aneurysm formation: early events and consequences of nitric oxide inhibition, Ann. Vasc. Surg. 16 (2002) 65-72.

[50] J.M. Johanning, D.P.M. Franklin, C. D, a. et, Inhibition of inducible nitric oxide synthase limits nitric oxide production and experimental aneurysm expansion, $\mathrm{J}$. Vasc. Surg. 33 (2001) 579-586.

[51] K.D. Kroncke, K. Fehsel, C. Suschek, V. Kolb-Bachofen, Inducible nitric oxide synthase-derived nitric oxide in gene regulation, cell death and cell survival, Int Immunopharmacol. 1 (2001) 1407-1420.

[52] D.C. Paik, W.G. Ramey, J. Dillon, M.D. Tilson, The nitrite/elastin reaction: implications for in vivo degenerative effects, Connect. Tissue Res. 36 (1997) 241-251.

[53] J. Zhang, J. Schmidt, E. Ryschich, M. Mueller-Schilling, H. Schumacher, J.R. Allenberg, Inducible nitric oxide synthase is present in human abdominal aortic aneurysm and promotes oxidative vascular injury, J. Vasc. Surg. 38 (2003) 360-367.

[54] S. Saito, N. Zempo, A. Yamashita, H. Takenaka, K. Fujioka, K. Esato, Matrix metalloproteinase expressions in arteriosclerotic aneurysmal disease, Vasc. Endovasc. Surg. 36 (2002) 1-7.

[55] E. Choke, G. Cockerill, W.R. Wilson, S. Sayed, J. Dawson, I. Loftus, M.M. Thompson, A review of biological factors implicated in abdominal aortic aneurysm rupture, Eur. J. Vasc. Endovasc. Surg. 30 (2005) 227-244.

[56] F. Aziz, H. Kuivaniemi, Role of matrix metalloproteinase inhibitors in preventing abdominal aortic aneurysm, Ann. Vasc. Surg. 21 (2007) 392-401.

[57] Z.S. Galis, M. Muszynski, G.K. Sukhova, E. Simon-Morrissey, E.N. Unemori, M.W. Lark, E. Amento, P. Libby, Cytokine-stimulated human vascular smooth muscle cells synthesize a complement of enzymes required for extracellular matrix digestion, Circ. Res. 75 (1994) 181-189.

[58] I.O. Peshkova, G. Schaefer, E.K. Koltsova, Atherosclerosis and aortic aneurysm - is inflammation a common denominator? FEBS J. 283 (2016) 1636-1652.

[59] N.L. Sehgel, Y. Zhu, Z. Sun, J.P. Trzeciakowski, Z. Hong, W.C. Hunter, D.E. Vatner, G.A. Meininger, S.F. Vatner, Increased vascular smooth muscle cell stiffness: a novel mechanism for aortic stiffness in hypertension, Am. J. Physiol. Heart Circ. Physiol. 305 (2013) H1281-H1289.

[60] H. Qiu, Y. Zhu, Z. Sun, J.P. Trzeciakowski, M. Gansner, C. Depre, R.R. Resuello, F.F. Natividad, W.C. Hunter, G.M. Genin, E.L. Elson, D.E. Vatner, G.A. Meininger, S.F. Vatner, Short communication: vascular smooth muscle cell stiffness as a mechanism for increased aortic stiffness with aging, Circ. Res. 107 (2010) 615-619.

[61] Z. Hong, K.J. Reeves, Z. Sun, Z. Li, N.J. Brown, G.A. Meininger, Vascular smooth muscle cell stiffness and adhesion to collagen I modified by vasoactive agonists, PLoS One 10 (2015) e0119533.

[62] E. Crosas-Molist, T. Meirelles, J. López-Luque, C. Serra-Peinado, J. Selva, L. Caja, D. Gorbenko, J.J. Uriarte, E. Bertran, Y. Mendizábal, V. Hernández, C. GarcíaCalero, O. Busnadiego, E. Condom, D. Toral, M. Castellà, A. Forteza, D. Navajas, E. Sarri, F. Rodríguez-Pascual, H.C. Dietz, I. Fabregat, G. Egea, Vascular smooth muscle cell phenotypic changes in patients with Marfan syndrome, Arterioscler. Thromb. Vasc. Biol. 35 (2015) 960-972.

[63] K. Komori, A. Tsujimura, T. Takao, Y. Matsuoka, Y. Miyagawa, S. Takada N. Nonomura, A. Okuyama, Nitric oxide synthesis leads to vascular endothelial growth factor synthesis via the $\mathrm{NO}$ /cyclic guanosine 3',5'-monophosphate (cGMP) pathway in human corpus cavernosal smooth muscle cells, J. Sex. Med. 5 (2008) 1623-1635.

[64] P. Ganz, P.F. Davies, J.A. Leopold, M.A. Gimbrone, R.W. Alexander, Short- and long-term interactions of endothelium and vascular smooth muscle in coculture: effects on cyclic GMP production, Proc. Natl. Acad. Sci. U. S. A. 83 (1986) 3552-3556.

[65] S. Fukumoto, H. Koyama, M. Hosoi, K. Yamakawa, S. Tanaka, H. Morii, Y. Nishizawa, Distinct role of cAMP and cGMP in the cell cycle control of vascular smooth muscle cells: cGMP delays cell cycle transition through suppression of cyclin D1 and cyclin-dependent kinase 4 activation, Circ. Res. 85 (1999) 985-991.

[66] M. Huffman, J. Curci, G. Moore, D. Kerns, B. Starcher, R. Thompson, Functional importance of connective tissue repair during the development of experimental abdominal aortic aneurysms, Surgery 128 (2000) 429-438.

[67] W.R. Wilson, M. Anderton, E.C. Schwalbe, J.L. Jones, P.N. Furness, P.R. Bell, M.M. Thompson, Matrix metalloproteinase- 8 and -9 are increased at the site of abdominal aortic aneurysm rupture, Circulation 113 (2006) 438-445.

[68] M.V. Gurjar, R.V. Sharma, R.C. Bhalla, eNOS gene transfer inhibits smooth muscle cell migration and MMP-2 and MMP-9 activity, Arterioscler. Thromb. Vasc. Biol. 19 (1999) 2871-2877.

[69] L.A. Ridnour, A.N. Windhausen, J.S. Isenberg, N. Yeung, D.D. Thomas, M.P. Vitek, D.D. Roberts, D.A. Wink, Nitric oxide regulates matrix metalloproteinase-9 activity by guanylyl-cyclase-dependent and -independent pathways, Proc. Natl. Acad. Sci. U. S. A. 104 (2007) 16898-16903. 